\title{
On the Apportionment of Population Structure
}

\author{
Yaron Granot ${ }^{1 *}$, Omri Tal $^{2}$, Saharon Rosset ${ }^{3}$, Karl Skorecki $^{1}$ \\ 1 Rappaport Faculty of Medicine and Research Institute, Technion-Israel Institute of Technology, and \\ Rambam Medical Center, Haifa, Israel, 2 Max Planck Institute for Mathematics in the Sciences, Inselstr. 22- \\ 26, 04103, Leipzig, Germany, 3 School of Mathematical Sciences Tel Aviv University, Tel Aviv, Israel \\ *yarongranot@ hotmail.com
}

\section{Abstract}

Measures of population differentiation, such as $\mathrm{F}_{\mathrm{ST}}$, are traditionally derived from the partition of diversity within and between populations. However, the emergence of population clusters from multilocus analysis is a function of genetic structure (departures from panmixia) rather than of diversity. If the populations are close to panmixia, slight differences between the mean pairwise distance within and between populations (low $\mathrm{F}_{\mathrm{ST}}$ ) can manifest as strong separation between the populations, thus population clusters are often evident even when the vast majority of diversity is partitioned within populations rather than between them. For any given $\mathrm{F}_{\mathrm{ST}}$ value, clusters can be tighter (more panmictic) or looser (more stratified), and in this respect higher $\mathrm{F}_{\mathrm{ST}}$ does not always imply stronger differentiation. In this study we propose a measure for the partition of structure, denoted $\mathrm{E}_{\mathrm{ST}}$, which is more consistent with results from clustering schemes. Crucially, our measure is based on a statistic of the data that is a good measure of internal structure, mimicking the information extracted by unsupervised clustering or dimensionality reduction schemes. To assess the utility of our metric, we ranked various human (HGDP) population pairs based on $\mathrm{F}_{\mathrm{ST}}$ and $E_{S T}$ and found substantial differences in ranking order. $E_{S T}$ ranking seems more consistent with population clustering and classification and possibly with geographic distance between populations. Thus, $E_{S T}$ may at times outperform $F_{S T}$ in identifying evolutionary significant differentiation.

\section{Introduction}

Genetic differentiation among populations is typically derived from the ratio of within- to between-population diversity. The most commonly used metric, $\mathrm{F}_{\mathrm{ST}}$, was originally introduced as a fixation index at a single biallelic locus [1], and subsequently adapted as a measure of population subdivision by averaging over multiple loci [2-3]. $\mathrm{F}_{\mathrm{ST}}$ can be expressed mathematically in terms of population diversities as $F_{S T}=1-S / T$, where $S$ and $T$ represent the heterozygosity in subpopulations and in the total population, respectively [4-5]. The validity of $\mathrm{F}_{\mathrm{ST}}$ as a measure of differentiation has been brought into question, especially when gene diversity is high (e.g., in 
microsatellites), and various metrics, including $\mathrm{G}_{\mathrm{ST}}^{\prime}[6]$ and Jost's $D$ [7], have been proposed to address this inadequacy (though see [8] for a counter-perspective).

Although these metrics vary considerably in their formulation, they all follow the same basic framework of partitioning genetic diversity into within- vs. between-group components. It has long been noted, however, that the apportionment of diversity [9] does not directly reflect the strength of separation between populations, and the emergence of population clusters has been demonstrated both empirically [10] and mathematically [5, 11-12] even when the vast majority of diversity is within rather than between populations. For example, humans sampled from across Europe [13] form identifiable clusters with pairwise $\mathrm{F}_{\mathrm{ST}}$ as low as 0.001 , even though $99.9 \%$ of the variation is contained within populations and only $0.1 \%$ is between them. Clearly, these clusters reflect an aspect of population differentiation that is not directly captured by $\mathrm{F}_{\mathrm{ST}}$, yet there is currently no commonly used metric for partitioning structure into within- and between-population components in the same way that $\mathrm{F}_{\text {ST }}$ partitions diversity. Dimensionality reduction schemes such as principal component analysis (PCA) [14] and clustering algorithms such as the widely used STRUCTURE [15] are highly popular, however such programs are primarily used in the population-genetics literature for visualization purposes, and there is still value in summary statistics for quantifying complex datasets on a simple $0-1$ scale.

Here we propose a novel measure, denoted $\mathrm{E}_{\mathrm{ST}}$, which is based on the variation in pairwise genetic distance (which we show to be a measure of internal structure), thus exposing the excess structure inherent in the total population compared to subpopulations. Conceptually, $\mathrm{E}_{\mathrm{ST}}$ is formulated in three steps: 1 . Population structure is defined in terms of departures from panmixia. 2 Panmixia is defined in terms of pairwise genetic equidistance between individuals (we show that a population is panmictic if all individuals are equally distant from each other). 3. Departures from equidistance are defined in terms of the standard deviation of pairwise distances. $\mathrm{E}_{\mathrm{ST}}$ reflects the decrease in panmixia when subpopulations are pooled. The basic formula is $\mathrm{E}_{\mathrm{ST}}=1-\mathrm{SD}_{\mathrm{S}} / \mathrm{SD}_{\mathrm{T}}$, where $\mathrm{SD}_{\mathrm{S}}$ and $\mathrm{SD}_{\mathrm{T}}$ represent the standard deviations of pairwise distances in subpopulations and in the total population respectively. While $\mathrm{F}_{\mathrm{ST}}$ is weighed down (diminished) by high diversity within populations, $\mathrm{E}_{\mathrm{ST}}$ is weighed down by high structure within populations.

A core insight of this proposal is that the asymptotic (in terms of number of genetic markers considered) standard deviation of pairwise genetic distances is a good unsupervised measure of internal structure, a statistic that mimics the information extracted by dimensionality reduction and clustering schemes, thus justifiable as a basis for the definition of $\mathrm{E}_{\mathrm{ST}}$. In particular, we show (in Appendix A) that a population is panmictic if and only if all individuals are asymptotically equally distant from each other, and that the standard deviation of pairwise genetic distances is highly associated with the deviation from panmixia and faithfully reflects the structure extracted by PCA.

\section{Results and Discussion}

\section{Partitioning Diversity vs. Partitioning Structure}

The difference between partitioning diversity and structure within and between two populations from the human genome diversity project (HGDP) [16] is illustrated in Fig 1. By zooming into a Russian and Chinese neighbor-joining tree of individual similarities we observe three layers of diversity and structure. Distances between individuals (black) account for most of the diversity, followed by the between-population component (red) and lastly, structure within populations (blue). The striking symmetry in the full-sized tree (1A) suggests high levels of panmixia in these two populations. Even at 100x magnification, most intra population branches (blue) are shorter than the $1 \mathrm{x}$ individual branches (black), indicating that these two 


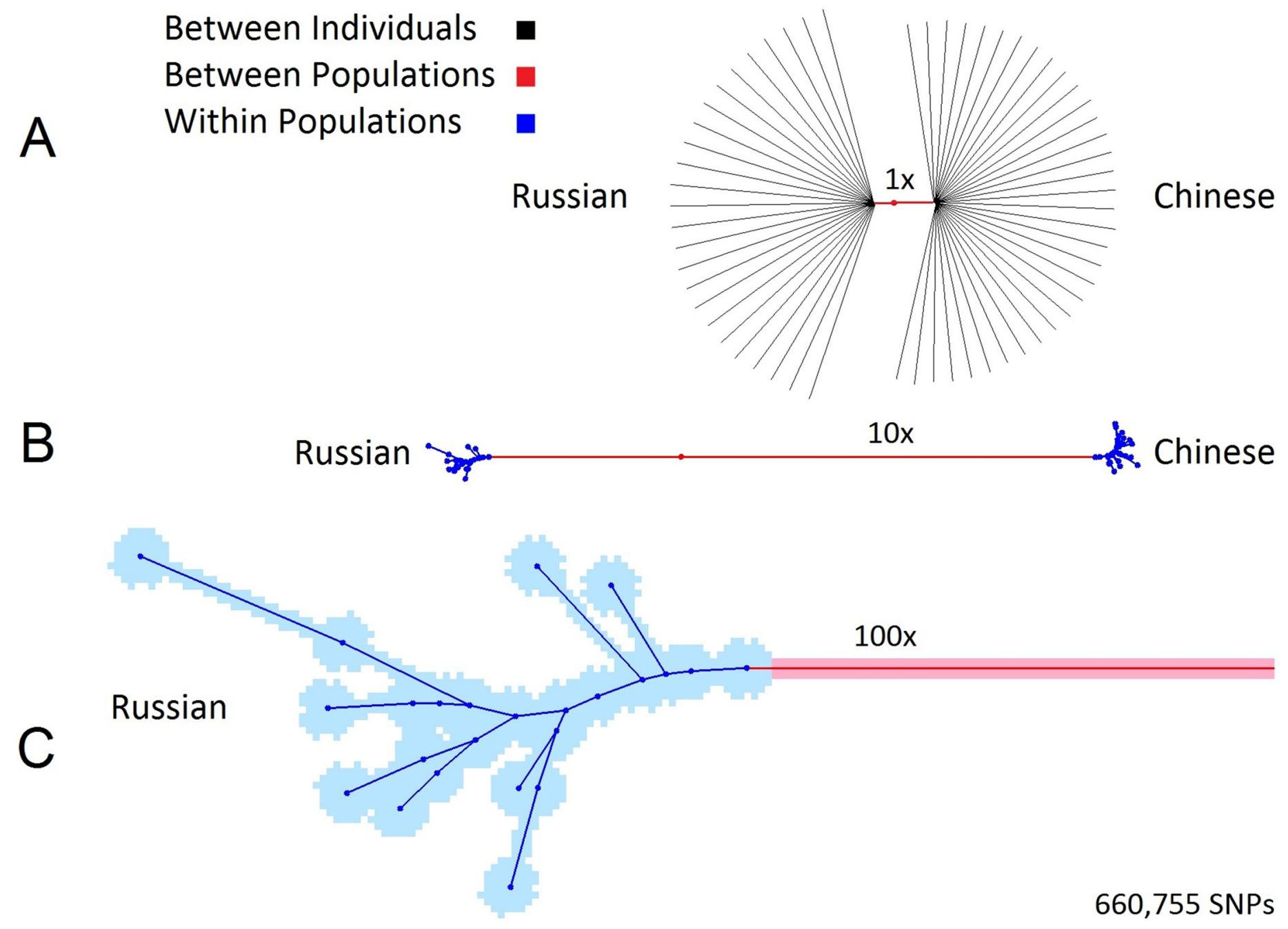

Fig 1. Zooming into a Russian $(n=25)$ and Chinese $(n=34)$ Neighbor Joining tree of individual similarities. (A) The length of the red branch compared to the overall tree length is a rough proxy to $\mathrm{F}_{\mathrm{ST}}$. (B) $10 \mathrm{x}$ magnification highlights the structure within and between populations. The blue branches inversely associate with $\mathrm{E}_{\mathrm{ST}}$ values. (C) 100x magnification reveals fine substructure within Russian samples. Individual branches (black) were removed in $\mathrm{B}$ and $\mathrm{C}$ for clarity.

doi:10.1371/journal.pone.0160413.g001 


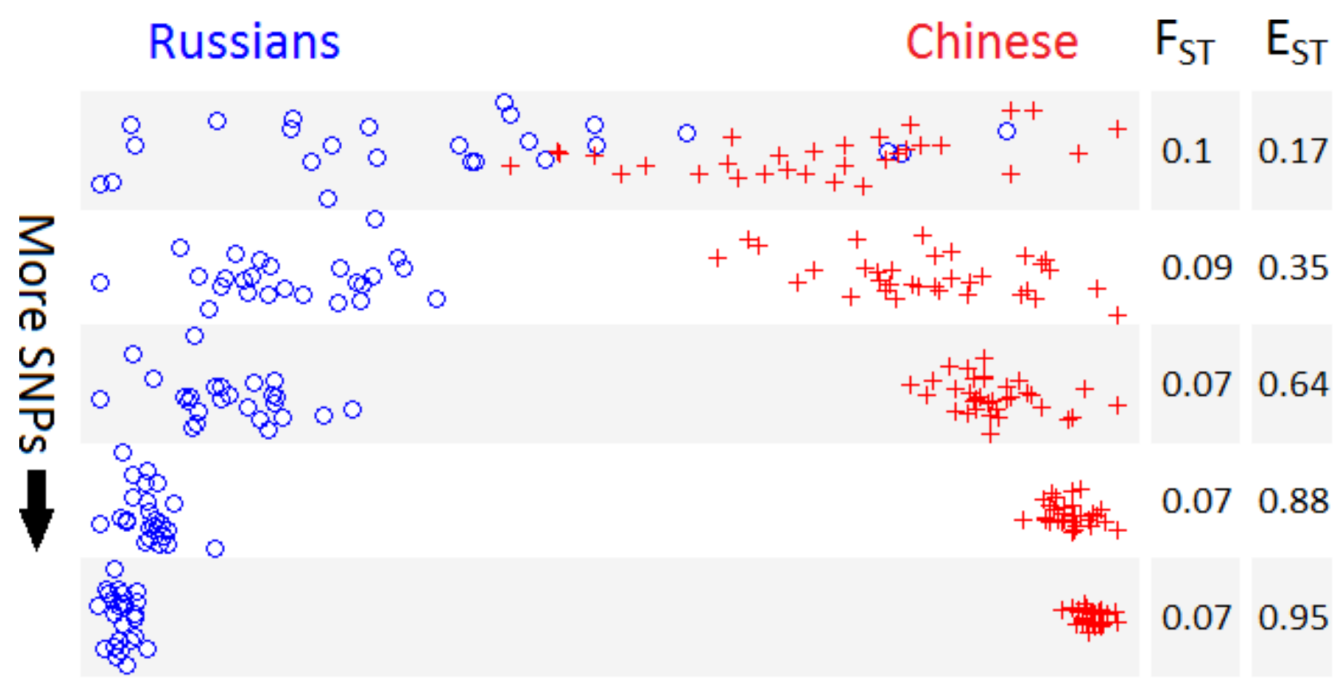

Fig 2. $F_{\mathrm{ST}}$ and $\mathrm{E}_{\mathrm{ST}}$ vs. Clustering with increasing SNP count. Multidimensional scaling (MDS) plots with Russian $(n=25)$ and Chinese $(n=34)$ samples with increasing SNP count from top to bottom $(10,100,1000$, 10,000 , and 660,755 SNPs). Two clusters gradually emerge as SNP count increases, along with an increase in $E_{S T}$, while $F_{S T}$ remains relatively constant.

doi:10.1371/journal.pone.0160413.g002

markers, and the amount of extractable information is further reduced by physical linkage. In our HGDP data the increase in $\mathrm{E}_{\mathrm{ST}}$ as a function of marker count reaches a plateau approximately above 100,000 SNPs (Fig 3). Although this upper bound can vary across different datasets and types of markers, it suggests that resolution may not improve substantially with further increases in marker count. Thus, these clusters can be considered close approximations of the "true" strength of separation among these populations. For this reason, when estimating $\mathrm{E}_{\mathrm{ST}}$ one should include as many markers as possible, although at a certain point additional markers provide greatly diminishing returns.

In order to determine whether or not $\mathrm{E}_{\mathrm{ST}}$ adds insight to the analysis of population structure, we sought to compare the rank order of population differentiation using $\mathrm{F}_{\mathrm{ST}}$ and $\mathrm{E}_{\mathrm{ST}}$. Pairwise $\mathrm{F}_{\mathrm{ST}}$ and $\mathrm{E}_{\mathrm{ST}}$ values from various HGDP populations are given in Table 1 (see $\mathrm{S} 1$ Table and S1, S5 Figs for additional comparisons). It is noted that for almost all population pairs $\mathrm{E}_{\mathrm{ST}}$ is larger than $\mathrm{F}_{\mathrm{ST}}$, and only the Colombian-Maya pair entails a slightly lower $\mathrm{E}_{\mathrm{ST}}$ than $\mathrm{F}_{\mathrm{ST}}$, presumably due to a combination of relatively low differentiation and high levels of intra-population structure. According to the HGDP browser (http://spsmart.cesga.es/search.php? dataSet=ceph_stanford, the Colombians $(\mathrm{n}=7)$ are the only HGDP population sample where two different tribes (Piapoco and Curripaco) were combined, which can help explain the high level of structure observed in this particular population (see S1 Table, S9, S5 Figs, and Materials and Methods for further analysis of $\mathrm{E}_{\mathrm{ST}}$ range). There is a moderate positive correlation $(\mathrm{r}=0.61)$ between $\mathrm{F}_{\mathrm{ST}}$ and $\mathrm{E}_{\mathrm{ST}}$ among all 60 population pairs included in our analysis (Fig 4), which is consistent with the two measures capturing somewhat different aspects of population structure. This seemingly high correlation can be attributed to the low (and therefore relatively similar) level of structure in many HGDP populations; the correlation between $\mathrm{E}_{\mathrm{ST}}$ and $\mathrm{F}_{\mathrm{ST}}$ tends to be higher when populations are more panmictic (see appendix).

\section{Amazonians vs. Global Populations}

A prominent example of the divergent behavior of $\mathrm{E}_{\mathrm{ST}}$ and $\mathrm{F}_{\mathrm{ST}}$ is the Surui and Karitiana, which have an unusually high pairwise $\mathrm{F}_{\mathrm{ST}}$. In fact, the Karitiana are as diverged from the 
..... Surui Karitiana

- - Russian Chinese

Russian Sardinian

n

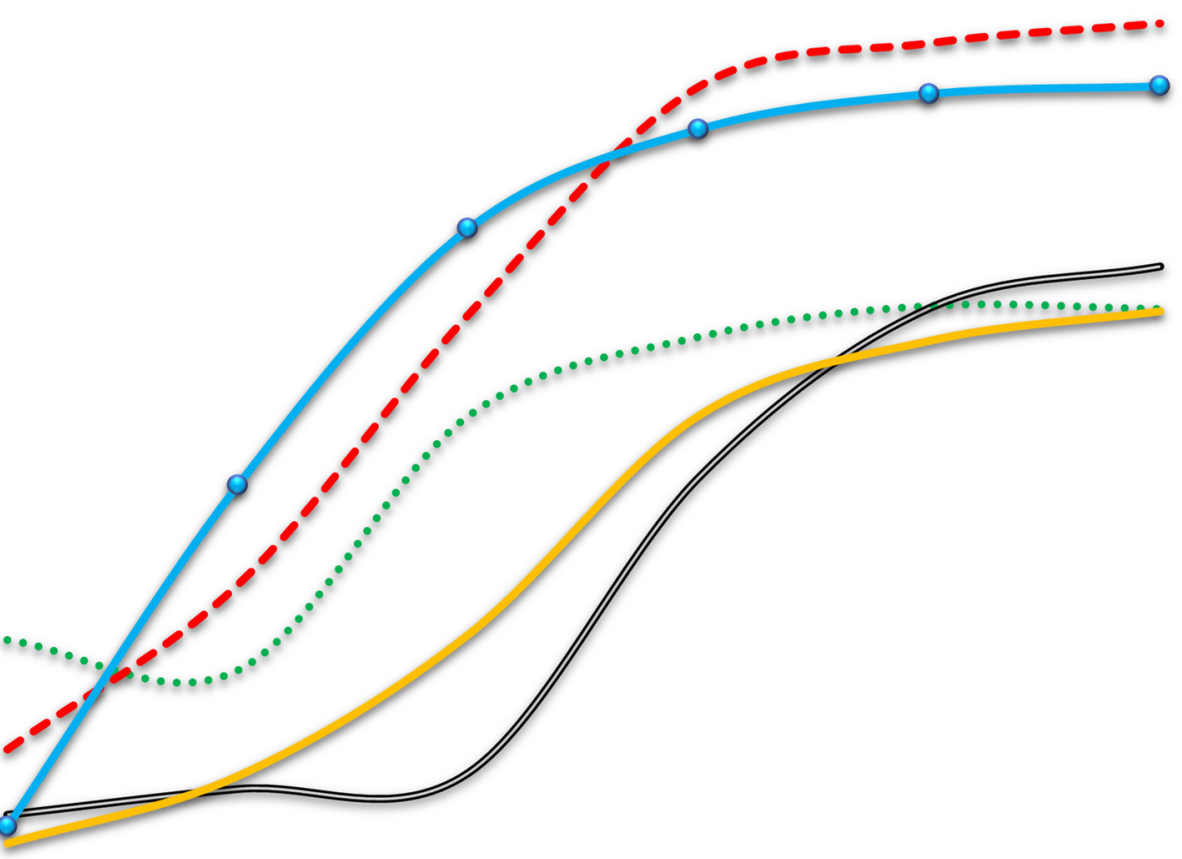

- Africa Europe

\begin{tabular}{lcccccc}
\cline { 2 - 7 } Number of SNPs & 10 & 100 & 1000 & 10000 & 100000 & 660755 \\
Surui Karitiana & 0.287402 & 0.255082 & 0.527655 & 0.612226 & 0.645473 & 0.642203 \\
Russian Chinese & 0.169798 & 0.346408 & 0.63601 & 0.880498 & 0.926977 & 0.948114 \\
Russian Sardinian & 0.099688 & 0.127379 & 0.144685 & 0.461724 & 0.64308 & 0.687639 \\
Balochi Kalash & 0.068614 & 0.141008 & 0.292738 & 0.527545 & 0.607674 & 0.639284 \\
Africa Europe & 0.086934 & 0.452973 & 0.727686 & 0.83431 & 0.872332 & 0.880404 \\
Mean & 0.1424872 & 0.26457 & 0.4657548 & 0.6632606 & 0.7391072 & 0.7595288 \\
Mean as \% of Max & $19 \%$ & $35 \%$ & $61 \%$ & $87 \%$ & $97 \%$ & $100 \%$
\end{tabular}

Fig 3. $E_{S T}$ as a function of SNP sample size. $E_{S T}$ was estimated in various population pairs with gradually increasing SNP sample size from 10 to 660,755 . As expected, $E_{S T}$ initially rises rather steeply, but tends to plateau before reaching the 660,755 SNP point. This suggests that we are approaching the maximal resolving power of genetic markers in this dataset, and adding markers beyond this point should not have a significant effect on -cluster separation and $\mathrm{E}_{\mathrm{ST}}$.

doi:10.1371/journal.pone.0160413.g003

neighboring Surui in terms of $\mathrm{F}_{\mathrm{ST}}$ as they are from the Mongola on the other side of the world (Table 1, Fig 5, and S5 Fig). F $_{\text {ST }}$ decreases initially with distance from the Amazon, from 0.13 between the two Amazonian tribes, to 0.08-0.1 between Amazonians and Colombians, and further decreases to $0.07-0.09$ between Amazonians and the more distant Maya. Remarkably, the highest $\mathrm{F}_{\mathrm{ST}}$ among all HGDP Native American populations is between the two geographically closest populations, the Surui and Karitiana. These apparent anomalies can be explained by the inflation of $\mathrm{F}_{\mathrm{ST}}$ among genetic isolates. $\mathrm{F}_{\mathrm{ST}}$ between pairs of isolates can be nearly twice as high as between either one of the isolates and a more cosmopolitan population, as pairwise 
Table 1. Pairwise $F_{S T}$ (above diagonal) and $E_{S T}$ (below diagonal) in 5 New World and 5 Old World HGDP populations.

\begin{tabular}{l|c|c|c|c|c|c|c|c|c|c|c}
\hline & Surui & Karitiana & Colombian & Maya & Pima & Yakut & Mongola & Russian & Bantu & San \\
\hline Surui & & 0.13 & 0.1 & 0.09 & 0.12 & 0.15 & 0.15 & 0.17 & 0.23 & 0.3 \\
\hline Karitiana & 0.58 & & 0.08 & 0.07 & 0.11 & 0.13 & 0.13 & 0.16 & 0.22 & 0.29 \\
\hline Colombian & 0.51 & 0.57 & & 0.03 & 0.06 & 0.09 & 0.09 & 0.12 & 0.18 & 0.25 \\
\hline Maya & 0.52 & 0.63 & 0.02 & & 0.04 & 0.07 & 0.06 & 0.08 & 0.15 & 0.21 \\
\hline Pima & 0.57 & 0.63 & 0.37 & 0.43 & & 0.1 & 0.09 & 0.12 & 0.19 & 0.25 \\
\hline Yakut & 0.74 & 0.8 & 0.6 & 0.69 & 0.74 & & 0.01 & 0.06 & 0.13 & 0.19 \\
\hline Mongola & 0.81 & 0.87 & 0.69 & 0.8 & 0.83 & 0.46 & & 0.06 & 0.12 & 0.19 \\
\hline Russian & 0.82 & 0.87 & 0.74 & 0.83 & 0.84 & 0.86 & 0.9 & 0.11 & 0.17 \\
\hline Bantu & 0.88 & 0.92 & 0.85 & 0.91 & 0.91 & 0.93 & 0.94 & 0.95 & 0.97 & 0.98 & 0.89
\end{tabular}

doi:10.1371/journal.pone.0160413.t001

$\mathrm{F}_{\mathrm{ST}}$ reflects the combined isolation of both populations. Since the Surui and Karitiana are both isolated, their pairwise $\mathrm{F}_{\mathrm{ST}}$ is nearly double that between any one of them and a larger, less isolated population such as the Maya.

Differentiation based on $\mathrm{E}_{\mathrm{ST}}$ (Surui-Karitiana $=0.58$, Karitiana-Mongola $=0.87$, and Mongola-Bantu $=0.94)$ seems more consistent with the geographic distances among these populations (Fig 5). It should be noted that the Surui-Karitiana $\mathrm{E}_{\mathrm{ST}}$ might be somewhat underestimated due to cryptic sampling of close relatives [17], however the wide range of

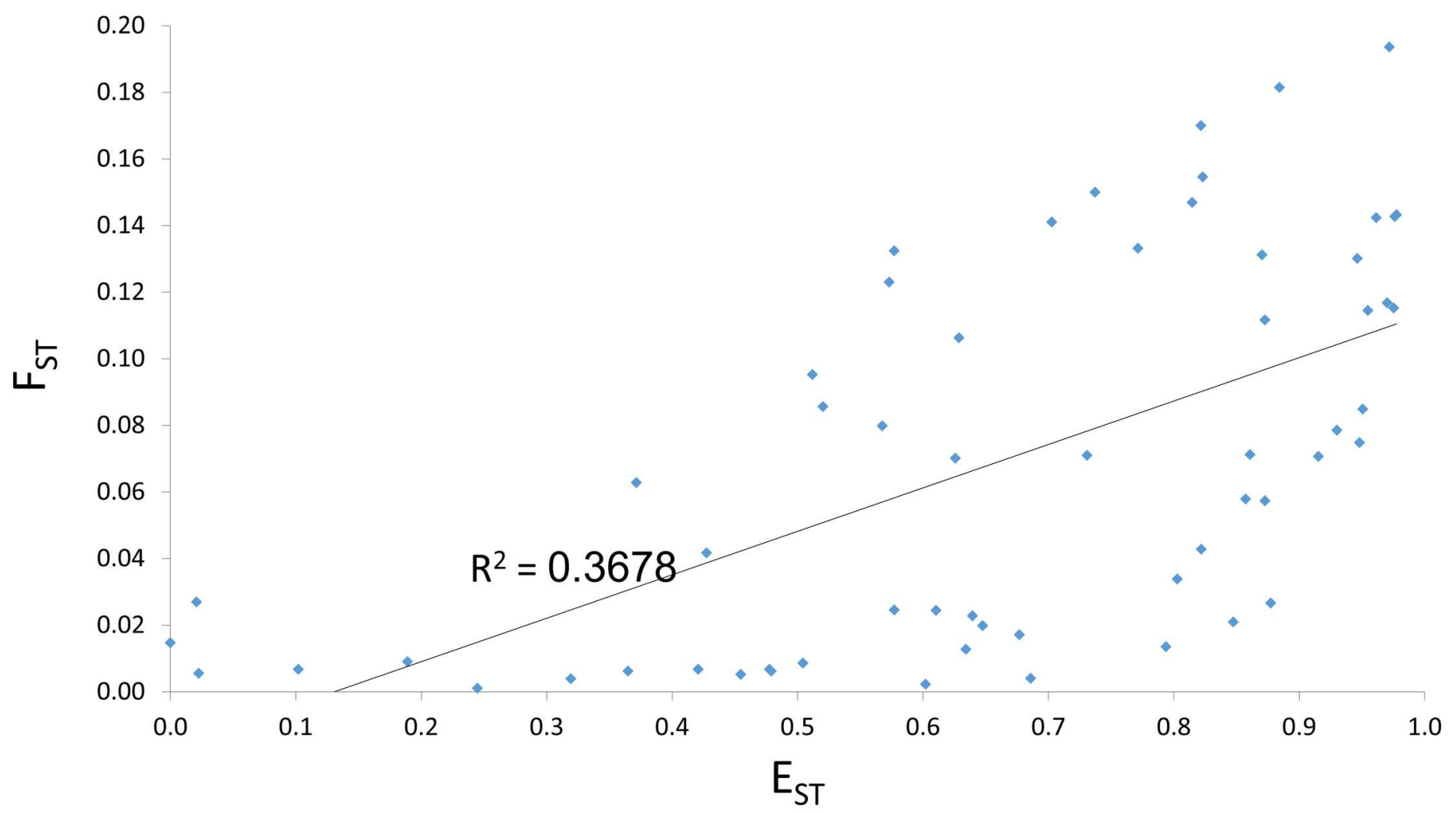

Fig 4. Positive correlation between $F_{S T}$ and $E_{S T} . R=0.61$. Note that $E_{S T}$ has a much broader range, spanning nearly the entire $0-1$ interval while $F_{S T}$ only goes as high as 0.2 in these HGDP populations.

doi:10.1371/jurnal.pone.0160413.g004 


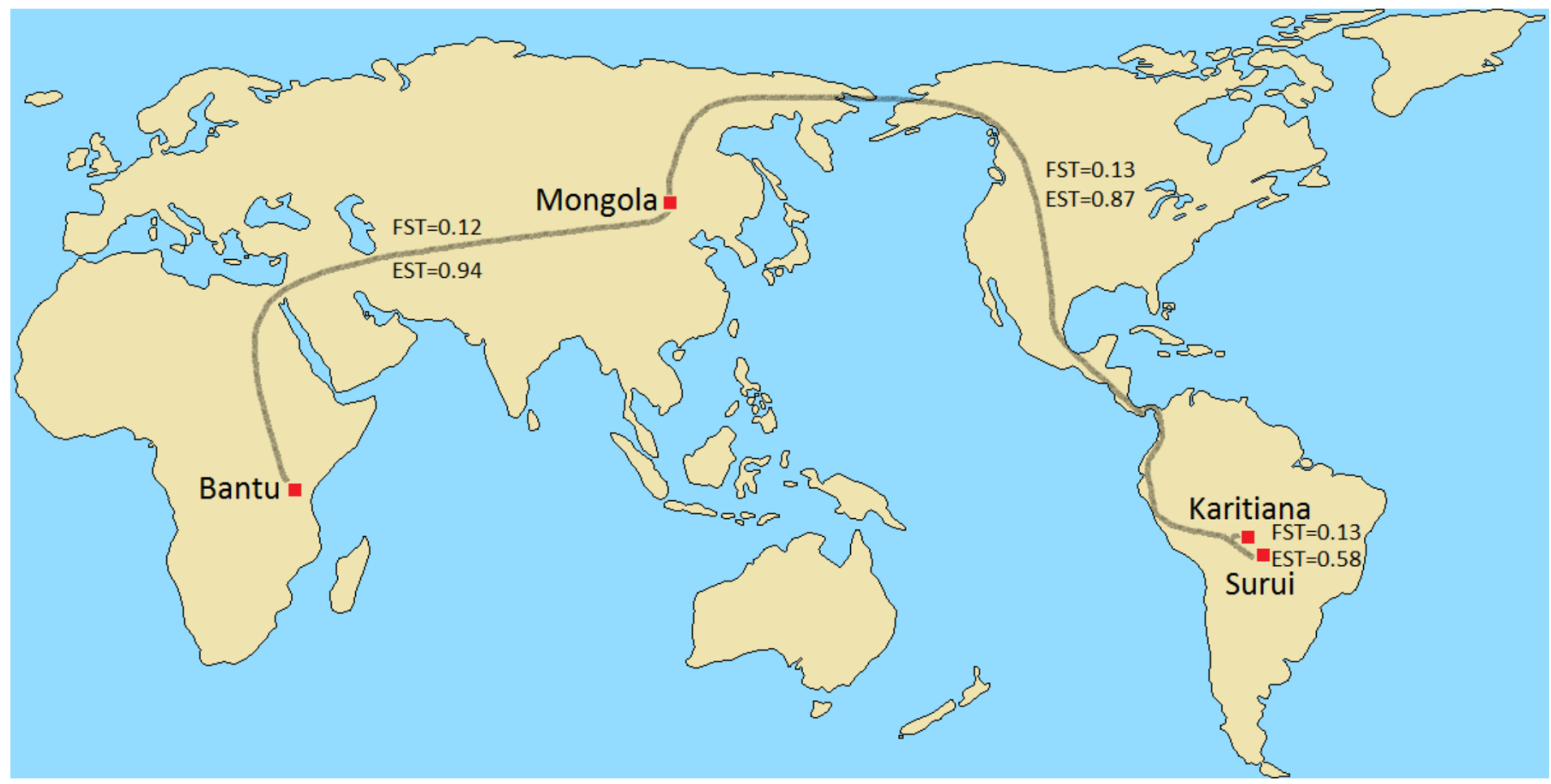

Fig 5. Geographic distance vs. $F_{S T}$ and $E_{S T}$ in various populations. In terms of $F_{S T}$, the Karitiana are roughly as diverged from the nearby $S$ urui $\left(F_{S T}=0.13\right)$ as they are from the Mongola on the other side of the world $\left(F_{S T}=0.13\right)$ or as the Bantu are from the Mongola $\left(F_{S T}=0.12\right)$. In terms of $E_{S T}$, differentiation is far greater among these global populations $\left(\mathrm{E}_{\mathrm{ST}} \approx 0.9\right)$ than between the neighboring Amazonian tribes $\left(\mathrm{E}_{\mathrm{ST}} \approx 0.6\right)$.

doi:10.1371/journal.pone.0160413.g005

heterozygosity values (which are less sensitive to the sampling of close relatives) and the elevated structure across all Native American HGDP populations (S3-S5 Figs) suggest that this is not merely a sampling artifact. In some cases $\mathrm{E}_{\mathrm{ST}}$ also decreases with distance from the Amazon (Table 1), however this decrease is more moderate than the decrease in $\mathrm{F}_{\mathrm{ST}}$ (S5 Fig).

Neighbor-joining trees of individual similarities [18] are a convenient tool for representing multidimensional genetic data on a two-dimensional plane, while simultaneously displaying distances within and between populations. Two pairs of such trees, for Surui-Karitiana and Yoruba-Russians, are given in Fig 6, and we can see that in both cases distances are greater between individuals (black branches) than between populations (red branches) (Fig 6A).

The ratio of within- to between-population distance is roughly equivalent in the two population pairs, however the Yoruba-Russian tree is significantly flatter, indicating greater panmixia within these two populations (also see S6-S7 Figs). Adding a third dimension of intrapopulation structure (blue branches) highlights this discrepancy (Fig 6B), which is further accentuated by removing the inter-individual component (Fig 6C) and stretching the YorubaRussian tree to match the level of structure observed in the Surui-Karitiana tree (Fig 6D). At first glance the Amazonian tribes, with their long population branches, appear to be as differentiated as the Yoruba are from the Russians. Upon closer inspection, however, the Yoruba and Russians appear more strongly diverged. The Amazonian tribes are highly structured not only between them, but also within them, resulting in distant, but loosely separated clusters. This aspect of population structure is not captured by $\mathrm{F}_{\mathrm{ST}}$, which is actually slightly higher between the Surui and Karitiana (0.13) than between Yoruba and Russians (0.12), but is revealed by the higher $\mathrm{E}_{\mathrm{ST}}$ between Yoruba and Russians (0.97) compared to the Surui and Karitiana (0.58). 
Surui Karitiana

A

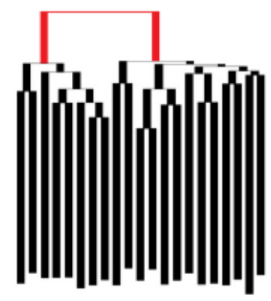

B

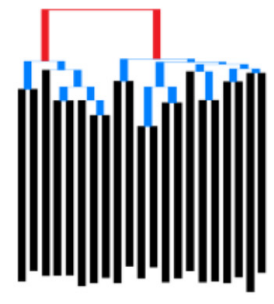

C

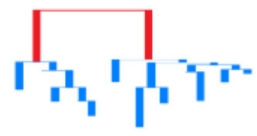

Yoruba

Russian
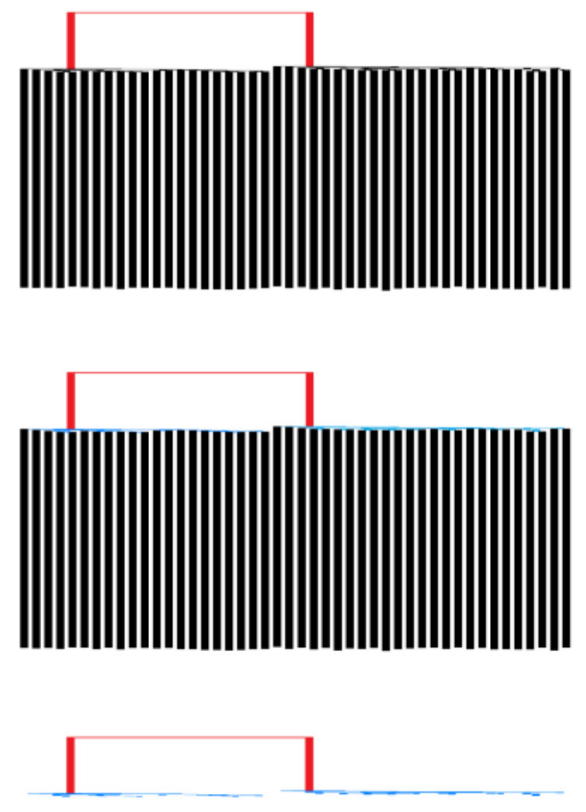

Between Individuals Between Populations Within Populations

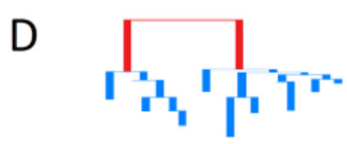

Surui Karitiana

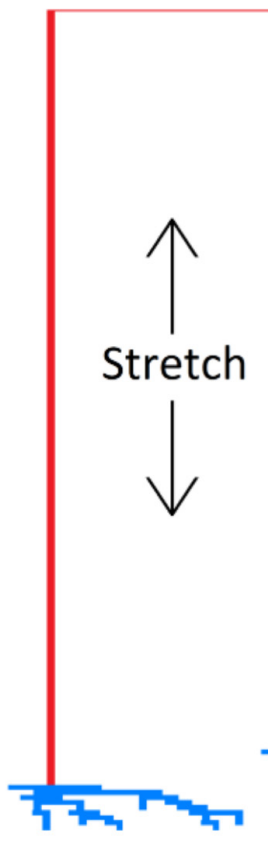

Yoruba

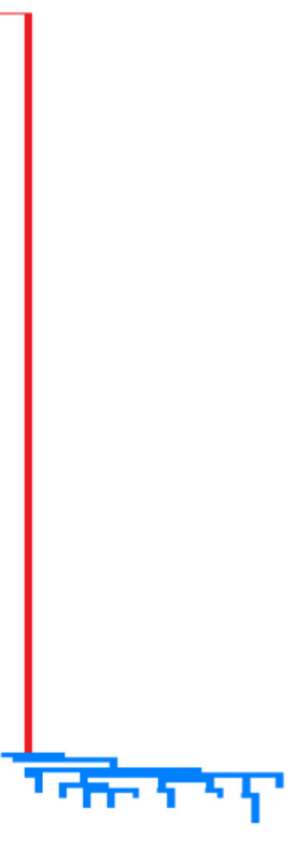

Russian

Fig 6. Surui-Karitiana vs. Yoruba-Russian NJ trees of individual similarities. (A) Diversity is apportioned into individual (black) and population (red) components. (B) A third component, structure within populations (blue), is added. (C) The individual component is removed. (D) The Yoruba-Russian tree is stretched to roughly match the level of structure within the Surui-Karitiana tree.

doi:10.1371/journal.pone.0160413.g006 


\section{$\mathrm{E}_{\mathrm{ST}}$ and the Dissimilarity Fraction}

Witherspoon et al. [10] have also examined population structure through the lens of pairwise genetic similarities and dissimilarities. They have defined the dissimilarity fraction, $\omega$, as the probability that individuals are genetically more similar to members of a different population than to members of their own population. An intuitive proxy for $\omega$ is (half) the overlap of the within and between pairwise distance distributions. For population pairs, this probability has a $0-0.5$ range, with the extremities $\omega=0$ indicating that individuals are always more similar to members of their own population and $\omega=0.5$ indicating that individuals are just as likely to be more similar to members of the other population as to members of their own population (see [5] for a formal analysis of such a metric and its relation to classification accuracy). Witherspoon et al. reported that that when many thousands of loci are analyzed, individuals from "geographically separated populations" are never more similar to each other than they are to members of their own populations. The definition of "geographically separated" is, of course, open to interpretation. We found no overlap $(\omega=0)$ between the Adygei and Uygur HGDP samples, but some overlap $(\omega>0)$ between Mayans and Surui, despite a $4 x$ higher $F_{\text {ST }}$ (Fig 7). Thus, $\mathrm{F}_{\mathrm{ST}}$ and the dissimilarity fraction $(\omega)$ are not necessarily congruent. The $\mathrm{E}_{\mathrm{ST}}$ values for these two population pairs are more consistent with $\omega$, showing strong separation between the Adygei and Uygur (0.79) and more moderate separation between Colombians and Maya (0.52) (see S8 Fig for a more detailed plot). The stronger association between $\omega$ and $\mathrm{E}_{\mathrm{ST}}$ is not surprising since both measures are sensitive to the presence of within-population structure (e.g., in the Maya and Surui), a property that is not captured by $\mathrm{F}_{\mathrm{ST}}$.
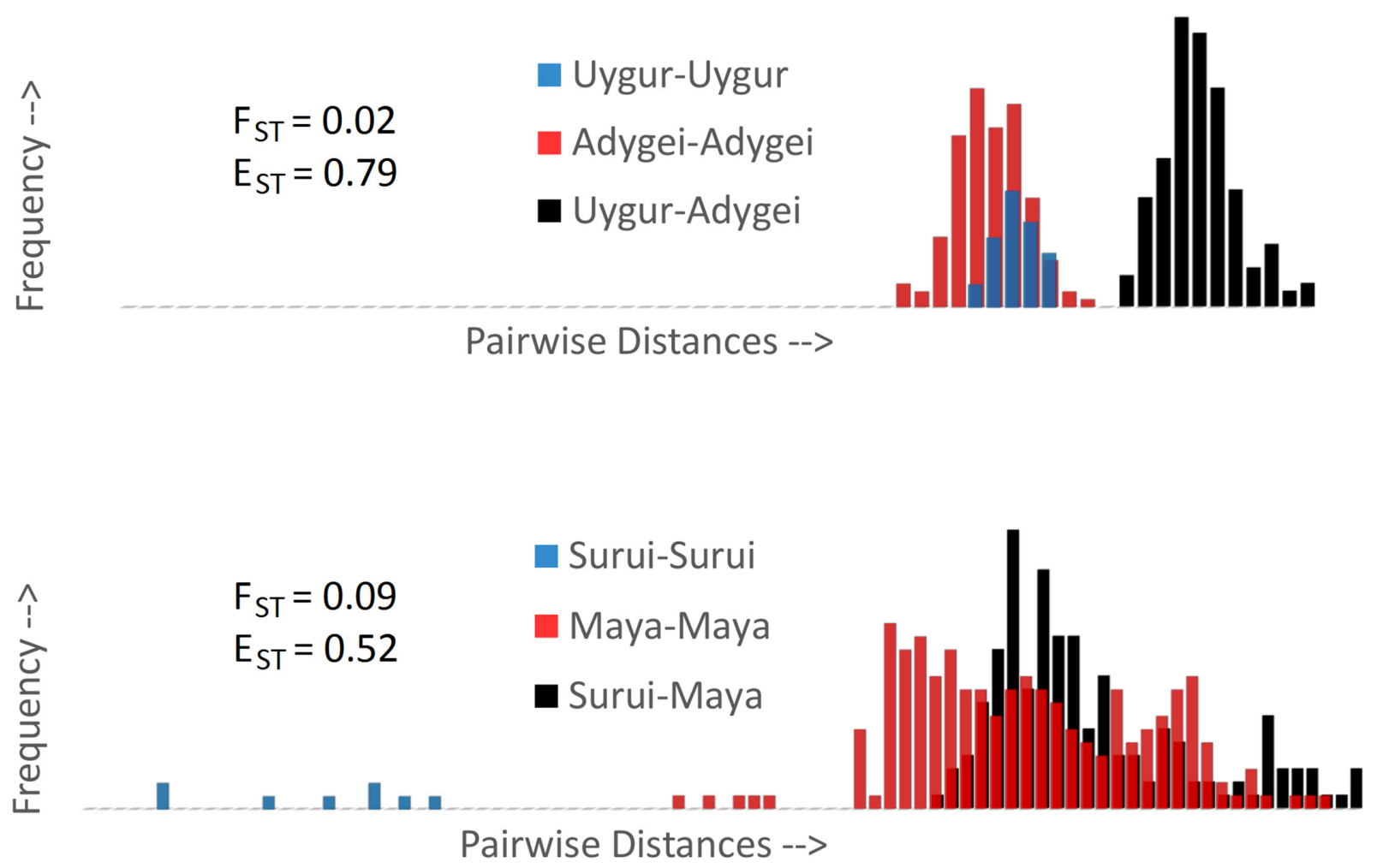

Fig 7. $F_{\mathbf{S T}}$ vs. genetic similarity in various population pairs. Pairwise distances are colored red or blue within populations and black between populations. (A) Even at a relatively low $F_{S T}$ of 0.02 all within-population pairs among the Uygur and Adygei samples are genetically more similar than all the between-population pairs. (B) Separation is more ambiguous among Native Americans. Despite a relatively high $\mathrm{F}_{\mathrm{ST}}$ of 0.09 , there is substantial overlap between Maya-Maya (red) and Maya-Surui (black) samples. $E_{S T}$ values are more consistent with the within- vs.-between population overlap and the dissimilarity fraction $(\omega)$.

doi:10.1371/journal.pone.0160413.g007 


\section{Summary and Conclusions}

The core distinction between $\mathrm{F}_{\mathrm{ST}}$ and $\mathrm{E}_{\mathrm{ST}}$ is that $\mathrm{F}_{\mathrm{ST}}$ partitions genetic diversity, whereas $\mathrm{E}_{\mathrm{ST}}$ partitions genetic structure within and between populations. While $\mathrm{F}_{\mathrm{ST}}$ is more sensitive to differences in within-population diversity, $\mathrm{E}_{\mathrm{ST}}$ is more sensitive to outliers (though this is largely mitigated by using $\mathrm{E}_{\mathrm{ST}}$ median rather than $\mathrm{E}_{\mathrm{ST}}$ mean; see Materials and Methods). Since $\mathrm{F}_{\mathrm{ST}}$ is weighed down by high levels of intrapopulation diversity, it can be close to zero even when population clusters are highly separated, however because it does not account for intrapopulation structure, high $\mathrm{F}_{\mathrm{ST}}$ does not necessarily reflect highly separated population clusters. This is not necessarily a flaw in $\mathrm{F}_{\mathrm{ST}}$, but it does demonstrate a conceptual discrepancy between $\mathrm{F}_{\mathrm{ST}}$ and strength of clustering.

Sewall Wright proposed a series of arbitrary $\mathrm{F}_{\mathrm{ST}}$ thresholds ranging from 0.05 to 0.25 , denoting little to very great differentiation [1]. Notably, the highest ranking of "very great differentiation" leaves most of the range (0.25-1) undefined. Given the wider empirical range of $\mathrm{E}_{\mathrm{ST}}$ and its correspondence with results from clustering schemes (Fig 2), phylogeography (Fig 5), and the dissimilarity fraction (Fig 7), such arbitrary thresholds may not be necessary for $\mathrm{E}_{\mathrm{ST}}$. A value of $\mathrm{E}_{\mathrm{ST}}$ larger than 0.5 simply indicates that most of the structure is between populations rather than within, corresponding to moderately separated populations such as Russians and Adygei $\left(\mathrm{E}_{\mathrm{ST}}=0.5\right)$, Bantu from South Africa and Kenya $\left(\mathrm{E}_{\mathrm{ST}}=0.48\right)$, or French and Sardinians $\left(\mathrm{E}_{\mathrm{ST}}=0.48\right)(\mathrm{S} 1 \mathrm{Table})$.

Differentiation metrics are judged by their ability to quantify meaningful evolutionary divergence, and can be indispensable in identifying Evolutionarily Significant Units (ESU) and Distinct Population Segments (DPS) for conservation [19]. For example, given several subpopulations within a species, it is reasonable to prioritize the most highly differentiated subpopulation for conservation in order to maximize biodiversity. However, higher $\mathrm{F}_{\mathrm{ST}}$ does not necessarily reflect stronger separation and lower misclassification, as with the Uygur and Adygei, whose clusters are better defined than those of the Surui and Maya despite a fourfold lower $\mathrm{F}_{\mathrm{ST}}$ (Fig 7). In this context humans can be a useful model species simply because we know so much about human populations due to our "long habit of observing ourselves" [20]. This allows us to make educated inferences about human populations that might otherwise be overlooked, e.g., we can be skeptical of the high Surui-Karitiana $\mathrm{F}_{\mathrm{ST}}$, and realize that this is most likely due to the relatively recent isolation of two small tribes. This is a luxury that we do not usually have with other species, in which case high $\mathrm{F}_{\mathrm{ST}}$ can be misinterpreted as a deep phylogenetic divide, potentially leading to misguided conservation strategies. Our hope is that by combining information from both fixation $\left(\mathrm{F}_{\mathrm{ST}}\right)$ and equidistance $\left(\mathrm{E}_{\mathrm{ST}}\right)$ indices, researchers could make more informed decisions.

Unlike $\mathrm{F}_{\mathrm{ST}}$, which is typically averaged across any number of markers, $\mathrm{E}_{\mathrm{ST}}$ is an asymptotic measure in the sense that it requires large datasets with many thousands of markers, which have only recently become widely accessible. With the latest SNP chips containing well over 100,000 markers, accurate estimates of departures from panmixia are finally within reach, and there is no longer a need for the simplifying assumption that subpopulations are effectively panmictic. By deriving an $\mathrm{F}_{\mathrm{ST}}$-type statistic for apportioning structure within and between populations, namely $\mathrm{E}_{\mathrm{ST}}$, we hope to add a new useful metric to the $21^{\text {st }}$ century population genetics toolkit.

\section{Materials and Methods}

The HGDP data used in our analysis were accessed at: http://www.hagsc.org/hgdp/files.html. After removing the 163 mitochondrial SNPs and 105 samples previously inferred to be close relatives [18], the final file included 660,755 SNPs from 938 samples in 53 populations. Strings 
of SNPs were treated as sequences, with mismatches summed and divided by the sequence length. Pairwise distances, based on Allele Sharing Distance (ASD) [21], were calculated as one minus half the average number of shared alleles per locus. The theoretical model, mathematical proofs and numerical simulations (using Mathematica v.8.0) of $\mathrm{SD}_{\mathrm{T}}$ and $\mathrm{SD}_{\mathrm{S}}$ appear in Appen$\operatorname{dix}$ A.

In the empirical analysis we used Hudson's pairwise-distance based $\mathrm{F}_{\mathrm{ST}}$ estimator [4] adapted to diploid genotypes:

$$
F_{S T}=1-\frac{S}{T}
$$

where $\mathrm{S}$ and $\mathrm{T}$ are mean pairwise distances within subpopulations and in the total pooled population.

$\mathrm{E}_{\mathrm{ST}}$ was formulated in terms of standard deviations as:

$$
E_{S T}=1-\frac{S D_{S}}{S D_{T}}
$$

where $\mathrm{SD}_{\mathrm{S}}$ and $\mathrm{SD}_{\mathrm{T}}$ are the standard deviations (SD) of pairwise distances within subpopulations and in the total population. This $\mathrm{E}_{\mathrm{ST}}$ estimator is referred to as $\mathrm{E}_{\mathrm{ST}}$ mean. We used three additional $\mathrm{E}_{\mathrm{ST}}$ estimators: $\mathrm{E}_{\mathrm{ST}} \mathrm{min}, \mathrm{E}_{\mathrm{ST}}$ median, and $\mathrm{E}_{\mathrm{ST}} \max$ ( $\mathrm{S} 9$ Fig). All four estimators use the same basic formula in $\mathrm{Eq}(2)$, with only the type of $\mathrm{SD}_{\mathrm{S}}$ differing among estimators. In $\mathrm{E}_{\mathrm{ST} \text { - }}$ min, $\mathrm{E}_{\mathrm{ST}}$ median, and $\mathrm{E}_{\mathrm{ST}} \mathrm{max}, \mathrm{SD}_{\mathrm{S}}$ is respectively replaced with the smallest, median, and largest individual SD, where the individual SD is the standard deviation of pairwise distances between a single sample and all other samples in the population. $\mathrm{E}_{\mathrm{ST}} \mathrm{min}$ uses the smallest individual $\mathrm{SD}_{\mathrm{S}}$ from each population, i.e., the $\mathrm{SD}$ of the most panmictic sample, $\mathrm{E}_{\mathrm{ST}}$ median uses the median individual $\mathrm{SD}_{\mathrm{S}}$, and $\mathrm{E}_{\mathrm{ST}} \max$ uses the highest individual $\mathrm{SD}_{\mathrm{S}}$. Each of these metrics has different sensitivities to various sampling biases. Due to $\mathrm{E}_{\mathrm{ST}}$ mean's sensitivity to the sampling of close relatives, we used $\mathrm{E}_{\mathrm{ST}}$ median (which is unaffected by the inclusion of relatives as long as at least $50 \%$ of the samples are unrelated) as the primary measure of $\mathrm{E}_{\mathrm{ST}}$ in this study. In the rare event that $>50 \%$ of the samples are closely related, $\mathrm{E}_{\mathrm{ST}}$ max may be preferable, as long as at last one individual has no close relatives among the samples. $\mathrm{E}_{\mathrm{ST}}$ values, especially $\mathrm{E}_{\mathrm{ST}} \mathrm{min}$ and $\mathrm{E}_{\mathrm{ST}}$ mean, can be negative if structure is high and differentiation is low (S9 Fig). Small sample sizes were often sufficient for estimating heterozygosity (S10 Fig) and $\mathrm{F}_{\mathrm{ST}}$ and $\mathrm{E}_{\mathrm{ST}}$ (S11 Fig) using all the SNPs in the HGDP dataset. Nevertheless, systematically developing estimators for $\mathrm{E}_{\mathrm{ST}}$ is beyond the scope of the current treatment.

We derived an additional equidistance index, denoted $\mathrm{E}_{\mathrm{BT}}$, which is less sensitive to intrapopulation structure and the inclusion of relatives. Recall that $\mathrm{E}_{\mathrm{ST}}$ reflects equidistance (E) within subpopulations (S) compared to the total (T) population. Similarly, $\mathrm{E}_{\mathrm{BT}}$ reflects equidistance (E) between subpopulations (B) compared to the total (T) population:

$$
E_{B T}=1-\frac{S D_{B}}{S D_{T}}
$$

Where $\mathrm{SD}_{\mathrm{B}}$ and $\mathrm{SD}_{\mathrm{T}}$ are the standard deviations of pairwise distances between individuals from different subpopulations, and in the total pooled population respectively. In most cases $\mathrm{SD}_{\mathrm{T}} \geq \mathrm{SD}_{\mathrm{B}}$, because $\mathrm{SD}_{\mathrm{T}}$ includes pairs of individuals from the same population as well as pairs from different populations, whereas $\mathrm{SD}_{\mathrm{B}}$ only includes pairs of individuals from different populations. Pairs of individuals from the same population are likely to have a higher SD due to relatives in the samples, which disrupt the panmixia (e.g., in the Naxi population, see S2-S4 Figs). Panmictic populations are not just equidistant among themselves; they are also equidistant towards each other. Such populations should have similar $\mathrm{SD}_{\mathrm{S}}$ and $\mathrm{SD}_{\mathrm{B}}$, and thus similar 
$\mathrm{E}_{\mathrm{ST}}$ and $\mathrm{E}_{\mathrm{BT}}$. Interestingly, some East Asians populations have relatively low $\mathrm{E}_{\mathrm{BT}}$, such as Cambodians vs. Mongola $\left(\mathrm{E}_{\mathrm{BT}}=0.13\right)$ and Japanese vs. Chinese $\left(\mathrm{E}_{\mathrm{BT}}=0.16\right)$. All $\mathrm{F}_{\mathrm{ST}}, \mathrm{E}_{\mathrm{ST}}$ and $\mathrm{E}_{\mathrm{BT}}$ estimates in this study are based on pairwise comparisons between two populations or population groups. Each of the two paired populations was given equal weight, as were the withinand between-population pairs. Thus, $25 \%$ of the total weight was given to each population, and $50 \%$ to between-population pairs.

We developed a custom MATLAB code for extracting genetic distances from SNP data and estimating heterozygosity, pairwise distances, $\mathrm{F}_{\mathrm{ST}}, \mathrm{E}_{\mathrm{ST}}$, and $\mathrm{E}_{\mathrm{BT}}$. The code corrects for missing data and small sample sizes, and identifies outliers, but includes no further assumptions or corrections. Phylogenetic trees and MDS plots were also generated with MATLAB. Equal angle and square neighbor-joining trees of individual similarities were generated from matrices of pairwise distances with the seqneighjoin command. An alternative script, based on the internal MATLAB seqpdist command for sequence distance, yielded similar results.

\section{Appendix A}

The standard deviation of pairwise distances as a measure of population structure

Our goal in this appendix is to substantiate the asymptotic (in terms of number of genetic markers) standard deviation of pairwise genetic distances as a good unsupervised measure of internal structure, thus justifiable as a basis for the definition of $E_{\mathrm{ST}}$. In particular, we prove that this asymptotic standard deviation is zero if and only if there is no internal structure (i.e., the population is panmictic).

\section{A model of pairwise genetic distances for genotypes from two diploid populations}

Let $p_{i}$ denote the frequency at locus $i$ of allele ' $A$ ' in population 1 , and let and $q_{i}$ denote the frequency of the same allele in population 2 and assume that both populations are effectively very large and have the same contribution to the total population. The commonly-used allele sharing distance (ASD) measures the dissimilarity of two individual genotypes. For diploid genotypes, it is commonly defined as 2 minus the number of shared alleles at each locus, averaged across loci [21-22]. For multiple loci genotypes we use a normalized (by the number of considered loci) version of ASD to simplify the analysis of means and variances of the ASD distribution, as in Tal (2013). Under the assumption of Hardy-Weinberg Equilibrium, allele frequencies fully determine per-locus genotype frequencies.

Let a categorical random variable $X_{i}$ represent the ASD at diploid locus $i$, and let $D_{n}$ represent the normalized ASD across $n$ loci for pairs of genotypes sampled from the total population,

$$
D_{n}=\frac{1}{n} \sum_{i=1}^{n} X_{i}
$$

We are interested in arriving at an expression for the variance (and ultimately the asymptotic standard deviation) of $D_{n}$. Given linkage equilibrium (LE) within each of our two subpopulations (required in order to allow modeling LE in the total population), the $X_{i}$ for the totalpopulation pairs are not statistically independent, and therefore the formulation for the variance of $D_{n}$ requires a partition into conditional expectations. From basic principles,

$$
\operatorname{Var}\left[D_{n}\right]=E\left[D_{n}^{2}\right]-E\left[D_{n}\right]^{2}
$$


Now, to evaluate $E\left[D_{n}^{2}\right]$ we need to condition it upon classification of pairs of genotypes as within- or between-population since the $X_{i}$ from the total population are not statistically independent,

$$
\begin{aligned}
E\left[D_{n}^{2}\right] & =E\left[\left(\frac{1}{n} \sum_{i=1}^{n} X_{i}\right)^{2}\right]=\frac{1}{n^{2}} E\left[\sum_{i=1}^{n} X_{i}^{2}+2 \sum_{i<j}^{n}\left(X_{i} \cdot X_{j}\right)\right]=\frac{1}{n^{2}}\left(\sum_{i=1}^{n} E\left[X_{i}^{2}\right]+2 \sum_{i<j}^{n} E\left[X_{i} \cdot X_{j}\right]\right) \\
& =\frac{1}{4} \cdot \frac{1}{n^{2}}\left(\sum_{i=1}^{n} E\left[X_{i}^{2}\right]+2 \sum_{i<j}^{n} E\left[X_{i}\right] \cdot E\left[X_{j}\right]\right) \mid \text { both genotypes from pop } 1 \\
& +\frac{1}{4} \cdot \frac{1}{n^{2}}\left(\sum_{i=1}^{n} E\left[X_{i}^{2}\right]+2 \sum_{i<j}^{n} E\left[X_{i}\right] \cdot E\left[X_{j}\right]\right) \mid \text { both genotypes from pop } 2 \\
& +\frac{1}{2} \cdot \frac{1}{n^{2}}\left(\sum_{i=1}^{n} E\left[X_{i}^{2}\right]+2 \sum_{i<j}^{n} E\left[X_{i}\right] \cdot E\left[X_{j}\right]\right) \mid \text { one genotype from pop } 1 \text { and one from } p o p 2
\end{aligned}
$$

where $E\left[X_{i} X_{j}\right]=E\left[x_{i}\right] \cdot E\left[X_{j}\right]$ since there is independence across any two loci for the within pairs and between pairs, and where the probabilities (assuming equal population sizes) for withinpopulation 1 pairs, within-population 2 pairs, and between-population pairs are at infinite population size $1 / 4,1 / 4,1 / 2$ respectively (otherwise, for finite population sizes $m$ we have probabilities $\frac{m-1}{4 m-2}, \frac{m-1}{4 m-2}, \frac{m}{2 m-1}$ respectively).

Now, from per-locus probabilities in Tal (2013, Eq 6 and Table 1) we derive the expected values,

$E\left[X_{i}\right] \mid$ both genotypes from pop $1=4\left(-p_{i}^{4}+2 p_{i}^{3}-2 p_{i}^{2}+p_{i}\right)$

$E\left[X_{i}^{2}\right] \mid$ both genotypes from pop $1=4 p_{i}\left(1-p_{i}\right)$

$E\left[X_{i}\right] \mid$ both genotypes from pop $2=4\left(-q_{i}^{4}+2 q_{i}^{3}-2 q_{i}^{2}+q_{i}\right)$

$E\left[X_{i}^{2}\right] \mid$ both genotypes from pop $2=4 q_{i}\left(1-q_{i}\right)$

$E\left[X_{i}\right] \mid$ one genotype from pop 1 and one from pop $2=2\left(2 p_{i} q_{i}^{2}+2 q_{i} p_{i}^{2}-2 p_{i}^{2} q_{i}^{2}-4 p_{i} q_{i}+p_{i}+q_{i}\right)$

$E\left[X_{i}^{2}\right] \mid$ one genotype from pop 1 and one from pop $2=2\left(p_{i}^{2}+q_{i}^{2}-4 p_{i} q_{i}+p_{i}+q_{i}\right)$

So that,

$$
\begin{aligned}
& E\left[D_{n}^{2}\right]=\frac{1}{n^{2}} \sum_{i=1}^{n} p_{i}\left(1-p_{i}\right)+8 \frac{1}{n^{2}} \sum_{i<j}^{n}\left(-p_{i}^{4}+2 p_{i}^{3}-2 p_{i}^{2}+p_{i}\right)\left(-p_{j}^{4}+2 p_{j}^{3}-2 p_{j}^{2}+p_{j}\right) \\
& +\frac{1}{n^{2}} \sum_{i=1}^{n} q_{i}\left(1-q_{i}\right)+8 \frac{1}{n^{2}} \sum_{i<j}^{n}\left(-q_{i}^{4}+2 q_{i}^{3}-2 q_{i}^{2}+q_{i}\right)\left(-q_{j}^{4}+2 q_{j}^{3}-2 q_{j}^{2}+q_{j}\right) \\
& +\frac{1}{n^{2}} \sum_{i=1}^{n}\left(p_{i}^{2}+q_{i}^{2}-4 p_{i} q_{i}+p_{i}+q_{i}\right) \\
& +4 \frac{1}{n^{2}} \sum_{i<j}^{n}\left(2 p_{i} q_{i}^{2}+2 q_{i} p_{i}^{2}-2 p_{i}^{2} q_{i}^{2}-4 p_{i} q_{i}+p_{i}+q_{i}\right)\left(2 p_{j} q_{j}^{2}+2 q_{j} p_{j}^{2}-2 p_{j}^{2} q_{j}^{2}-4 p_{j} q_{j}+p_{j}+q_{j}\right)
\end{aligned}
$$


Also, since the expectation of a sum of dependent random variables is the sum of their expectations we have for the 'total population' $X_{i}$,

$$
E\left[D_{n}\right]^{2}=\left(E\left[\frac{1}{n} \sum_{i=1}^{n} X_{i}\right]\right)^{2}=\frac{1}{n^{2}}\left(\sum_{i=1}^{n} E\left[X_{i}\right]\right)^{2}
$$

From Tal (2013, section 3.2) we have the expression for the pmf of $X_{i}$ and thus can derive $\mathrm{E}$ $\left[X_{i}\right]$

$$
\begin{aligned}
& E\left[X_{i}\right]=0 \cdot \operatorname{Pr}\left(X_{i}=0\right)+1 \cdot \operatorname{Pr}\left(X_{i}=1\right)+2 \cdot \operatorname{Pr}\left(X_{i}=2\right) \\
& =\frac{1}{4}\left(\left(p_{i}+q_{i}\right)^{3}\left(2-p_{i}-q_{i}\right)+\left(2-p_{i}-q_{i}\right)^{3}\left(p_{i}+q_{i}\right)+\left(p_{i}+q_{i}\right)^{2}\left(2-p_{i}-q_{i}\right)^{2}\right) \\
& =\frac{1}{4}\left(p_{i}+q_{i}\right)\left(2-p_{i}-q_{i}\right)\left(\left(p_{i}+q_{i}\right)^{2}+\left(2-p_{i}-q_{i}\right)^{2}+\left(p_{i}+q_{i}\right)\left(2-p_{i}-q_{i}\right)\right) \\
& =\frac{1}{4}\left(p_{i}+q_{i}\right)\left(2-p_{i}-q_{i}\right)\left(4-\left(p_{i}+q_{i}\right)\left(2-p_{i}-q_{i}\right)\right)
\end{aligned}
$$

Such that,

$$
E\left[D_{n}\right]^{2}=\frac{1}{16 n^{2}}\left(\sum_{i=1}^{n}\left(p_{i}+q_{i}\right)\left(2-p_{i}-q_{i}\right)\left(4-\left(p_{i}+q_{i}\right)\left(2-p_{i}-q_{i}\right)\right)\right)^{2}
$$

So that finally,

$$
\begin{aligned}
\operatorname{Var}\left[D_{n}\right]=E\left[D_{n}^{2}\right]-E\left[D_{n}\right]^{2} & =\frac{1}{n^{2}}\left[\sum_{i=1}^{n} p_{i}\left(1-p_{i}\right)+8 \sum_{i<j}^{n}\left(-p_{i}^{4}+2 p_{i}^{3}-2 p_{i}^{2}+p_{i}\right)\left(-p_{j}^{4}+2 p_{j}^{3}-2 p_{j}^{2}+p_{j}\right)\right. \\
& +\sum_{i=1}^{n} q_{i}\left(1-q_{i}\right)+8 \sum_{i<j}^{n}\left(-q_{i}^{4}+2 q_{i}^{3}-2 q_{i}^{2}+q_{i}\right)\left(-q_{j}^{4}+2 q_{j}^{3}-2 q_{j}^{2}+q_{j}\right) \\
& +\sum_{i=1}^{n}\left(p_{i}^{2}+q_{i}^{2}-4 p_{i} q_{i}+p_{i}+q_{i}\right) \\
& +4 \sum_{i<j}^{n}\left(2 p_{i} q_{i}^{2}+2 q_{i} p_{i}^{2}-2 p_{i}^{2} q_{i}^{2}-4 p_{i} q_{i}+p_{i}+q_{i}\right)\left(2 p_{j} q_{j}^{2}+2 q_{j} p_{j}^{2}-2 p_{j}^{2} q_{j}^{2}-4 p_{j} q_{j}+p_{j}+q_{j}\right) \\
& \left.-\frac{1}{16}\left(\sum_{i=1}^{n}\left(p_{i}+q_{i}\right)\left(2-p_{i}-q_{i}\right)\left(4-\left(p_{i}+q_{i}\right)\left(2-p_{i}-q_{i}\right)\right)\right)^{2}\right]
\end{aligned}
$$

Thus we have an explicit formulation for the variance of the pairwise distance distribution of genotypes from two panmictic populations in terms of the allele frequencies across a given number of loci, $n$.

Crucially, we would like to prove that at the limit, the pairwise distance variance is asymptotically above zero if and only if the population has internal structure; i.e., if for any $F_{\mathrm{ST}}>0$,

$$
S=\lim _{n \rightarrow \infty} \operatorname{Var}\left[D_{n}\right]>0
$$

We will proceed by deriving an explicit expression for $S$. Consider an equivalent setting comprised of three random variables $W, Y$ and $Z$, which represent the pairwise distances of genotypes within population 1 , within population 2 and between populations 1 and 2 , respectively. We sample $n$ values $X_{i}$ from just one of these distributions, by first flipping a 3-sided coin to decide from which: with a probability $\alpha$ for $W$, a probability $\beta$ for $Y$ and a probability $\gamma$ for $Z$. Once the distribution has been selected, the sampling of $X_{i}$ is done i.i.d. Note that due to 
the randomized choice of the distribution from which to sample all the $X_{i}$, they are identically distributed but not independent. Now we set,

$$
D_{n}=\frac{1}{n} \sum_{i=1}^{n} X_{i}
$$

We would like to get an expression for $S$ in terms of the expectations of $W, Y, Z$ and $\alpha, \beta, \gamma$, where,

$$
S=\lim _{n \rightarrow \infty} \operatorname{Var}\left(D_{n}\right)
$$

From the law of total variance,

$$
\operatorname{Var}\left(D_{n}\right)=\operatorname{Var}\left(E\left[D_{n} \mid B\right]\right)+E\left[\operatorname{Var}\left(D_{n} \mid B\right)\right]=\operatorname{Var}\left(U_{X Y Z}\right)+\frac{1}{n} C \cdot \operatorname{Var}\left(D_{n}\right)
$$

where $B$ is a categorical random variable that describes which of the distributions $W, Y, Z$ we are sampling from, with probabilities $\alpha, \beta, \gamma$ respectively, where $U_{X Y Z}$ is a discrete random variable taking the values of $\mu_{W}=E[W], \mu_{Y}=E[Y], \mu_{Z}=E[Z]$ with corresponding probabilities $\alpha$, $\beta, \gamma$ respectively, and where $\mathrm{C}$ is some constant. Hence at the limit $n \rightarrow \infty$ we have,

$$
\begin{gathered}
S=\operatorname{Var}\left(U_{X Y Z}\right)=\alpha\left(\mu_{W}-\mu\right)^{2}+\beta\left(\mu_{Y}-\mu\right)^{2}+\gamma\left(\mu_{Z}-\mu\right)^{2} \\
\mu=\alpha \mu_{W}+\beta \mu_{Y}+\gamma \mu_{Z}
\end{gathered}
$$

and $S=0$ if and only if the three means are equal, i.e., $\mu_{W}=\mu_{Y}=\mu_{Z}$.

Now consider three sequences of random variables $W_{i}, Y_{i}, Z_{i}, i: 1 \ldots n$, instead of the three single random variables, and sample $n$ values from one of these sequences (again according to the prior probabilities $\alpha, \beta, \gamma)$. Once the sequence is selected, these samples are independent but now not identically distributed. We would again like to find $S$, and more importantly, the condition for which it is zero, this time in terms of $E\left[W_{i}\right], E\left[Y_{i}\right], E\left[Z_{i}\right]$ (and the prior probabilities). Sampling from a sequence with fixed probabilities just defines a new mixture distribution-s o the problem gets reduced to the one already solved. Therefore $U_{X Y Z}$ is now defined by the three limits (since we have derived $S$ in Eq (8) at the limit $n \rightarrow \infty$ ),

$$
\mu_{W}=\lim _{n \rightarrow \infty} \frac{1}{n} \sum_{i=1}^{n} E\left[W_{i}\right], \mu_{Y}=\lim _{n \rightarrow \infty} \frac{1}{n} \sum_{i=1}^{n} E\left[Y_{i}\right], \mu_{Z}=\lim _{n \rightarrow \infty} \frac{1}{n} \sum_{i=1}^{n} E\left[Z_{i}\right]
$$

with probabilities $\alpha, \beta, \gamma$.

Crucially, this sampling scenario corresponds to our original setting of formulating the variance of the genetic distance of genotypes sampled from the total population, given the sequencing of an infinite number of loci, where $\mu_{W}$ and $\mu_{Y}$ in Eq (9) represent the two withinpopulation pairwise distance means and $\mu_{Z}$ the total-population mean (derived below), and where the respective probabilities are as in Eq (4), $\alpha=1 / 4, \beta=1 / 4, \gamma=1 / 2$, assuming infinite population size. Again, $S=0$ if and only if these means are equal, i.e., $\mu_{W}=\mu_{Y}=\mu_{Z}$. Let us analyze the conditions for these equalities, given the corresponding formulations of the pairwise distance means. First, using the additivity of expectations,

$$
E\left[D_{n}\right]=\frac{1}{n} \sum_{i=1}^{n} E\left[X_{i}\right]
$$


we get from $\mathrm{Eq}(5)$ the expressions for any finite $n$,

$E\left[D_{n}\right] \mid$ both genotypes from pop $1=\frac{1}{n} \sum_{i=1}^{n} 4\left(-p_{i}^{4}+2 p_{i}^{3}-2 p_{i}^{2}+p_{i}\right)$
$E\left[D_{n}\right] \mid$ both genotypes from pop $2=\frac{1}{n} \sum_{i=1}^{n} 4\left(-q_{i}^{4}+2 q_{i}^{3}-2 q_{i}^{2}+q_{i}\right)$

$E\left[D_{n}\right] \mid$ one genotype from pop 1 and one from pop $2=\frac{1}{n} \sum_{i=1}^{n} 2\left(2 p_{i} q_{i}^{2}+2 q_{i} p_{i}^{2}-2 p_{i}^{2} q_{i}^{2}-4 p_{i} q_{i}+p_{i}+q_{i}\right)$

bearing in mind the analysis pertains to $E\left[D_{n}\right]$ as $n \rightarrow \infty$. We proceed to examine what can be concluded from the equalities $\mu_{W}=\mu_{Y}=\mu_{Z}$ (the only case where $S=0$ ), given the means in Eq (10), about the allele frequencies $p_{i}$ and $q_{i}$ for any finite $n$ (and this also holds at $n \rightarrow \infty$ ). Thus we start by explicitly writing the equalities (where the $1 / n$ cancels out),

$$
\begin{aligned}
& \sum_{i=1}^{n}\left(-p_{i}^{4}+2 p_{i}^{3}-2 p_{i}^{2}+p_{i}\right)=\sum_{i=1}^{n}\left(-q_{i}^{4}+2 q_{i}^{3}-2 q_{i}^{2}+q_{i}\right) \\
& \sum_{i=1}^{n}\left(-p_{i}^{4}+2 p_{i}^{3}-2 p_{i}^{2}+p_{i}\right)=\sum_{i=1}^{n}\left(2 p_{i} q_{i}^{2}+2 q_{i} p_{i}^{2}-2 p_{i}^{2} q_{i}^{2}-4 p_{i} q_{i}+p_{i}+q_{i}\right)
\end{aligned}
$$

To proceed we substitute new variables,

$$
\begin{aligned}
& x_{i}=p_{i}\left(1-p_{i}\right) \\
& y_{i}=q_{i}\left(1-q_{i}\right)
\end{aligned}
$$

Then, the 1 st equation in (8) becomes,

$$
\sum_{i=1}^{n} x_{i}\left(1-x_{i}\right)=\sum_{i=1}^{n} y_{i}\left(1-y_{i}\right)
$$

such that,

$$
\sum_{i=1}^{n}\left[x_{i}\left(1-x_{i}\right)+y_{i}\left(1-y_{i}\right)\right]=\sum_{i=1}^{n} 2 x_{i}\left(1-x_{i}\right)
$$

and using the 2 nd equation in (8),

$$
\begin{aligned}
& =\sum_{i=1}^{n}\left[2 p_{i} q_{i}\left(q_{i}+p_{i}-p_{i} q_{i}-1\right)+p_{i}+q_{i}-2 p_{i} q_{i}\right] \\
& =\sum_{i=1}^{n}\left[-2 p_{i} q_{i}\left(1-p_{i}\right)\left(1-q_{i}\right)+\left(p_{i}-p_{i}^{2}\right)+\left(q_{i}-q_{i}^{2}\right)+\left(p_{i}^{2}+q_{i}^{2}-2 p_{i} q_{i}\right)\right]
\end{aligned}
$$

And again in terms of the new variables,

$$
\sum_{i=1}^{n}\left[-2 x_{i} y_{i}+x_{i}+y_{i}+\left(p_{i}-q_{i}\right)^{2}\right]
$$


A

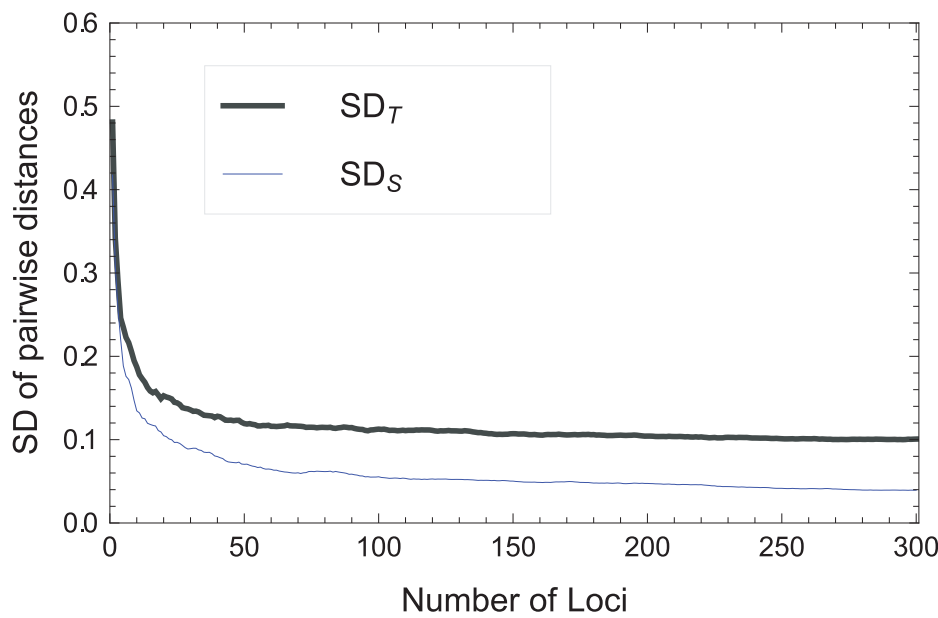

B

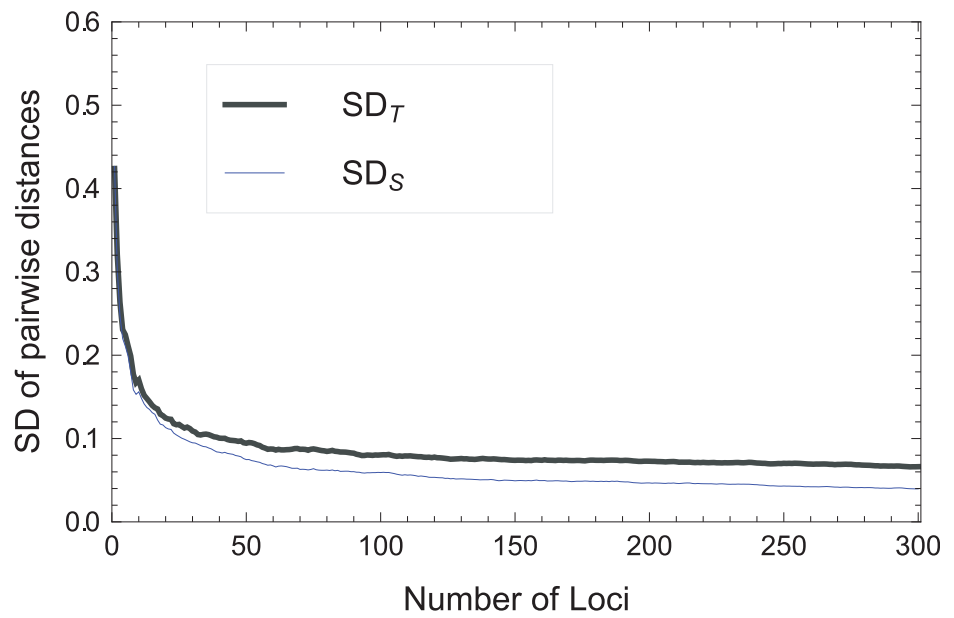

Fig 8. A simulation of $S D_{\mathrm{T}}$ and $S D_{\mathrm{S}}$ under a two panmictic population model demonstrating the divergent behavior of these two statistics with an increasing number of SNP loci. SNP frequencies are modeled on Beta distributions (as in [5]). (A) with $F_{\mathrm{ST}}=0.10$. (B) with $F_{\mathrm{ST}}=0.03$.

doi:10.1371/journal.pone.0160413.g008

This implies that,

$$
\sum_{i=1}^{n}\left[\left(x_{i}-y_{i}\right)^{2}+\left(p_{i}-q_{i}\right)^{2}\right]=0
$$

which occurs only if $p_{i}=q_{i}$ for all $i=1, \ldots, n$.

Therefore the asymptotic variance of the pairwise genetic distances (normalized by number of loci) of genotypes sampled from the combined population, comprising two subpopulations, is zero if this combined population is essentially a single panmictic population (i.e., $p_{i}=q_{i}$ for all $i$, or $F_{\mathrm{ST}}=0$ ). Since we have defined $E_{\mathrm{ST}}$ in terms of standard deviations rather than variances, we will subsequently consider the asymptotic standard deviation $S D_{\mathrm{T}}$, which is simply defined as the square root of the asymptotic variance, $S$ for the 'total' population. Fig 8 depicts numerical simulations of both $S D_{\mathrm{T}}$ and the average within-population $\mathrm{SD}\left(S D_{\mathrm{S}}\right)$ for our two population model, as a function of the number of $S N P s$ considered. While $S D_{S}$ converges to zero, $S D_{\mathrm{T}}$ asymptotes to a value greater than zero, revealing the underlying structure. We note here that the rate of convergence to zero for $S D_{\mathrm{S}}$ is highly dependent on the diversity of each population-for lower diversity the within-population SD converges faster (and thus tends to be 
lower for any finite number of loci). This factor also influences the rate of convergence of $E_{\mathrm{ST}}$ to its asymptotic value (see main text, Fig 3 ).

To further substantiate $S D_{\mathrm{T}}$ as a measure of structure, we would like to characterize the relation of $S D_{\mathrm{T}}$ to $F_{\mathrm{ST}}$, both formulated as expressions of allele frequencies from two populations. We will proceed numerically, as our goal here is merely to get a qualitative intuition into the association of the two statistics.

We have from Eqs (8), (9) and (10), that asymptotically as $n \rightarrow \infty$, or practically under a high number of SNP loci,

$$
S D_{T}=\sqrt{S}=\sqrt{\frac{1}{4}\left(\mu_{W}-\mu\right)^{2}+\frac{1}{4}\left(\mu_{Y}-\mu\right)^{2}+\frac{1}{2}\left(\mu_{Z}-\mu\right)^{2}}
$$

where,

$$
\begin{gathered}
\mu=\frac{1}{4} \mu_{W}+\frac{1}{4} \mu_{Y}+\frac{1}{2} \mu_{Z} \\
\mu_{W}=\lim _{n \rightarrow \infty} \frac{1}{n} \sum_{i=1}^{n} 4\left(-p_{i}^{4}+2 p_{i}^{3}-2 p_{i}^{2}+p_{i}\right) \\
\mu_{Y}=\lim _{n \rightarrow \infty} \frac{1}{n} \sum_{i=1}^{n} 4\left(-q_{i}^{4}+2 q_{i}^{3}-2 q_{i}^{2}+q_{i}\right) \\
\mu_{Z}=\lim _{n \rightarrow \infty} \frac{1}{n} \sum_{i=1}^{n} 2\left(2 p_{i} q_{i}^{2}+2 q_{i} p_{i}^{2}-2 p_{i}^{2} q_{i}^{2}-4 p_{i} q_{i}+p_{i}+q_{i}\right)
\end{gathered}
$$

And from Tal (2013, Eq 13) we use the most common expression for $F_{\mathrm{ST}}$ across any number of $n$ SNPs,

$$
F_{S T}=\frac{\sum_{i=1}^{n}\left(p_{i}-q_{i}\right)^{2}}{\sum_{i=1}^{n}\left(p_{i}+q_{i}\right)\left(2-p_{i}-q_{i}\right)}
$$

Under the standard assumption that SNP frequencies are modeled on a Beta distribution with parameters deriving from some historical process (see $[5,22]$ ) we sample a large number of sets of SNP frequencies for two subpopulations, each set generated from two Beta distributions with some randomized parameters. For each set we compute the pair $S D_{\mathrm{T}}$ (Eq 12) and $F_{\mathrm{ST}}$ (Eq 13) to generate a scatter plot of their association. Fig 9A-9D depicts several typical instances of such a simulation, demonstrating that the correlation of the two statistics is substantial when the total population is comprised of panmictic subpopulations,

$$
0<<\rho\left[S D_{T}, F_{S T}\right]<1
$$

and is lower if the subpopulations have varying degrees of structure. Extending the numerical analysis for any number of panmictic multiple populations indicates that $S D_{\mathrm{T}}$ closely follows $F_{\mathrm{ST}}$ as a measure of structure. 

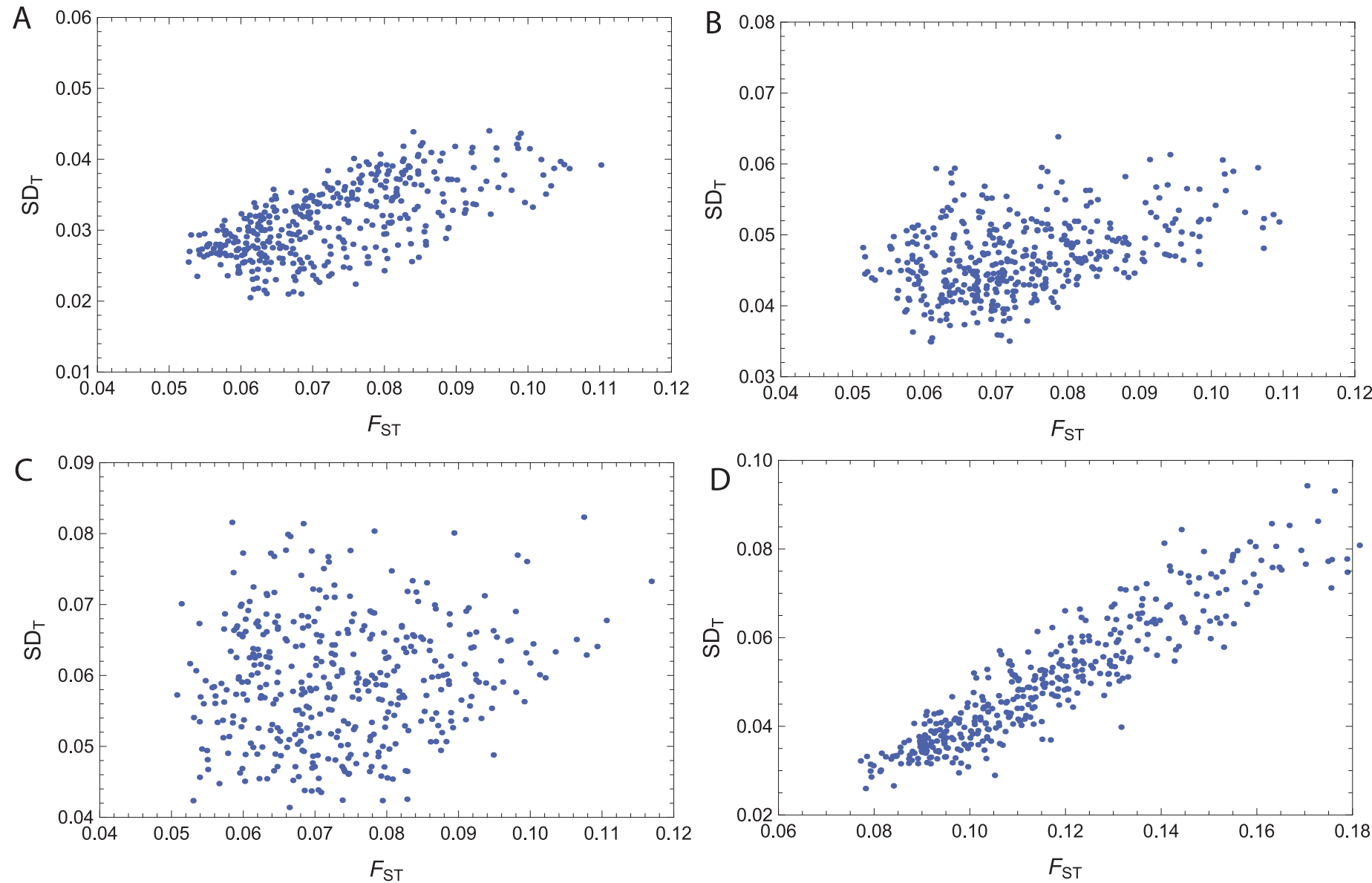

Fig 9. Scatter plots indicating a positive correlation for $S D_{\mathrm{T}}$ and $\boldsymbol{F}_{\mathrm{ST}}$. Each dot represents the two statistics computed for data sampled from our population model with 1000 SNPs and allele frequencies from Beta distributions. The Pearson product-moment correlation coefficient of $F_{S T}$ and $S D_{T}$ is 0.67 for plot (A) with panmictic populations, 0.38 for plot $(B)$ with slightly varying structure in subpopulations, 0.14 for plot $(C)$ with highly varying structure in subpopulations, and for 0.94 for plot (D) with three panmictic populations.

doi:10.1371/journal.pone.0160413.g009

We may generalize this result to more than two populations in a straightforward manner using induction. For instance, for three populations Eq (12) becomes,

$$
S D_{T}=\sqrt{\frac{1}{9}\left(\mu_{W 1}-\mu\right)^{2}+\frac{1}{9}\left(\mu_{W 2}-\mu\right)^{2}+\frac{1}{9}\left(\mu_{W 3}-\mu\right)^{2}+\frac{2}{9}\left(\mu_{B 1}-\mu\right)^{2}+\frac{2}{9}\left(\mu_{B 2}-\mu\right)^{2}+\frac{2}{9}\left(\mu_{B 3}-\mu\right)^{2}(14}
$$

where,

$$
\begin{gathered}
\mu=\frac{1}{9} \mu_{W 1}+\frac{1}{9} \mu_{W 2}+\frac{1}{9} \mu_{W 3}+\frac{2}{9} \mu_{B 1}+\frac{2}{9} \mu_{B 2}+\frac{2}{9} \mu_{B 3} \\
\mu_{W 1}=\lim _{n \rightarrow \infty} \frac{1}{n} \sum_{i=1}^{n} 4\left(-p_{i}^{4}+2 p_{i}^{3}-2 p_{i}^{2}+p_{i}\right) \\
\mu_{W 2}=\lim _{n \rightarrow \infty} \frac{1}{n} \sum_{i=1}^{n} 4\left(-q_{i}^{4}+2 q_{i}^{3}-2 q_{i}^{2}+q_{i}\right)
\end{gathered}
$$



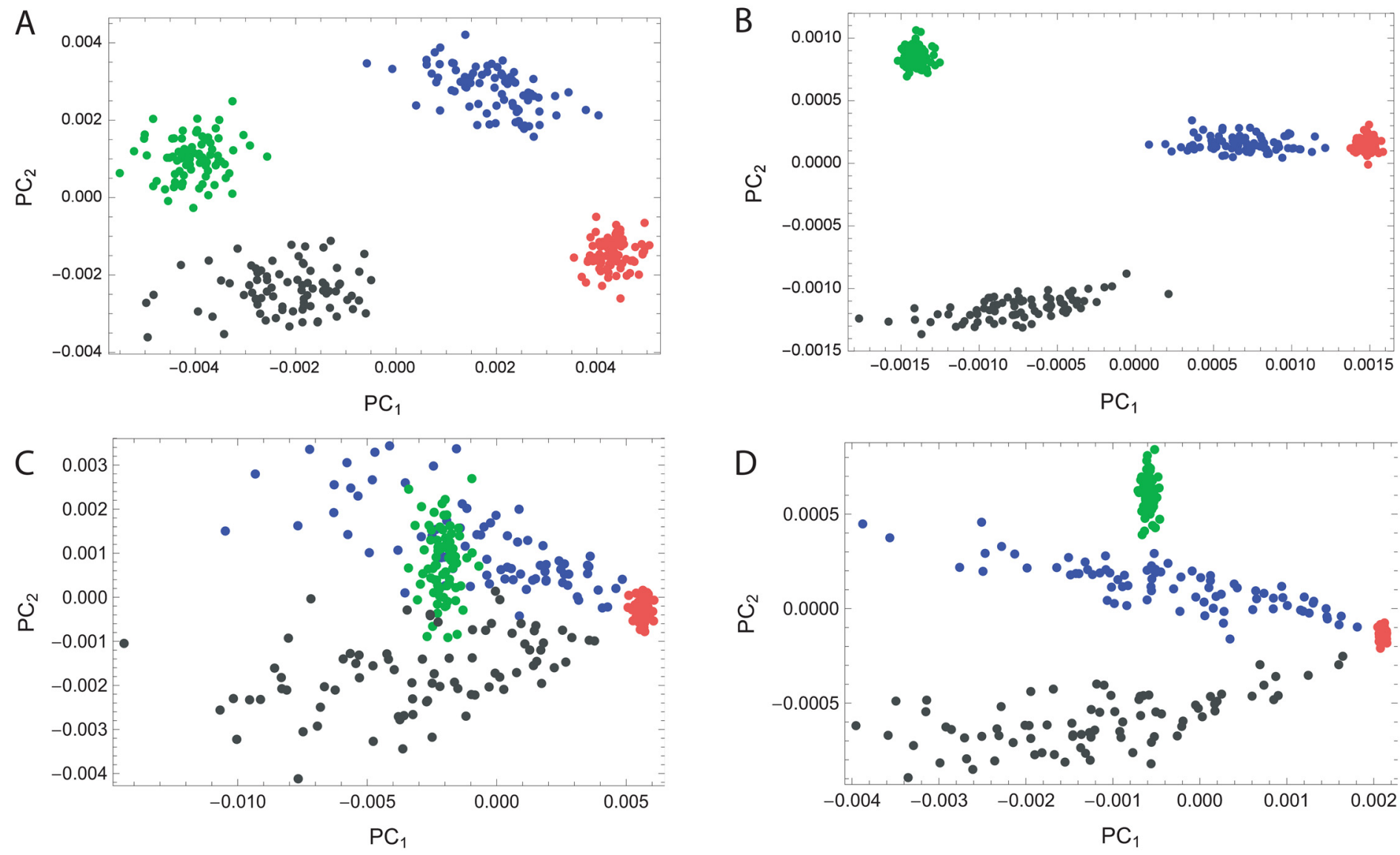

Fig 10. PCA plots from simulated SNP data of four populations (80 samples each) demonstrating the much pronounced decrease in $S D_{S}$ for panmictic populations (red and green) relative to structured ones (black and blue), for two different patterns of internal structure, as the number of SNP loci processed by the PCA scheme is increased. (A-B) from 1K SNPs to 8K SNPs, where structure results from some random linkage disequilibrium (LD) pattern. (C-D) from 1K SNPs to 8K SNPs, where structure results from an LD pattern resembling admixture.

doi:10.1371/journal.pone.0160413.g010

$$
\begin{gathered}
\mu_{W 3}=\lim _{n \rightarrow \infty} \frac{1}{n} \sum_{i=1}^{n} 4\left(-r_{i}^{4}+2 r_{i}^{3}-2 r_{i}^{2}+r_{i}\right) \\
\mu_{B 1}=\lim _{n \rightarrow \infty} \frac{1}{n} \sum_{i=1}^{n} 2\left(2 p_{i} q_{i}^{2}+2 q_{i} p_{i}^{2}-2 p_{i}^{2} q_{i}^{2}-4 p_{i} q_{i}+p_{i}+q_{i}\right) \\
\mu_{B 2}=\lim _{n \rightarrow \infty} \frac{1}{n} \sum_{i=1}^{n} 2\left(2 p_{i} r_{i}^{2}+2 r_{i} p_{i}^{2}-2 p_{i}^{2} r_{i}^{2}-4 p_{i} r_{i}+p_{i}+r_{i}\right) \\
\mu_{B 3}=\lim _{n \rightarrow \infty} \frac{1}{n} \sum_{i=1}^{n} 2\left(2 r_{i} q_{i}^{2}+2 q_{i} r_{i}^{2}-2 r_{i}^{2} q_{i}^{2}-4 r_{i} q_{i}+r_{i}+q_{i}\right)
\end{gathered}
$$

and where $F_{\mathrm{ST}}$ for 3 panmictic populations with SNP loci is derived in the same manner as 
Eq (13),

$$
F_{S T}=\frac{\sum_{i=1}^{n} 2\left(p_{i}^{2}+q_{i}^{2}+r_{i}^{2}-p_{i} q_{i}-p_{i} r_{i}-r_{i} q_{i}\right)}{\sum_{i=1}^{n}\left(p_{i}+q_{i}+r_{i}\right)\left(3-p_{i}-q_{i}-r_{i}\right)}
$$

A further perspective into $S D_{\mathrm{T}}$ as an unsupervised measure of internal structure is afforded by a qualitative comparison with principal component analysis (PCA) plots on data generated by the model. PCA is an unsupervised technique, essentially a dimensionality reduction procedure, used to emphasize the directions of greatest variation and bring out any strong patterns in a dataset. It can be used as a 'preprocessing' stage for clustering high-dimensional data, such as characteristic of population genetic samples. In such a setting, the first principal components tend to also extract existing substructure within the data in the form of clusters [14]. But more crucially to our goals, the relative dispersion of clusters on a PCA plot is highly associated with their internal structure, i.e., departures from panmixia, with increasing number of loci (and asymptotically, panmictic clusters would diminish to a single dot). This property is congruent with the convergence of $S D_{\mathrm{T}}$ to some value strictly greater than zero for non-panmictic populations. This is depicted in the four PCA plots of the same populations under increasing SNP count in Fig 10A-10D.

Finally, through numerical simulation of our model, we can see how varying degrees of internal structure (simulated by controlling the LD patterns) result in different asymptotic levels of $E_{\mathrm{ST}}$ (Fig 11). This serves to substantiate the empirical analysis depicted in Fig 3 of the main text.

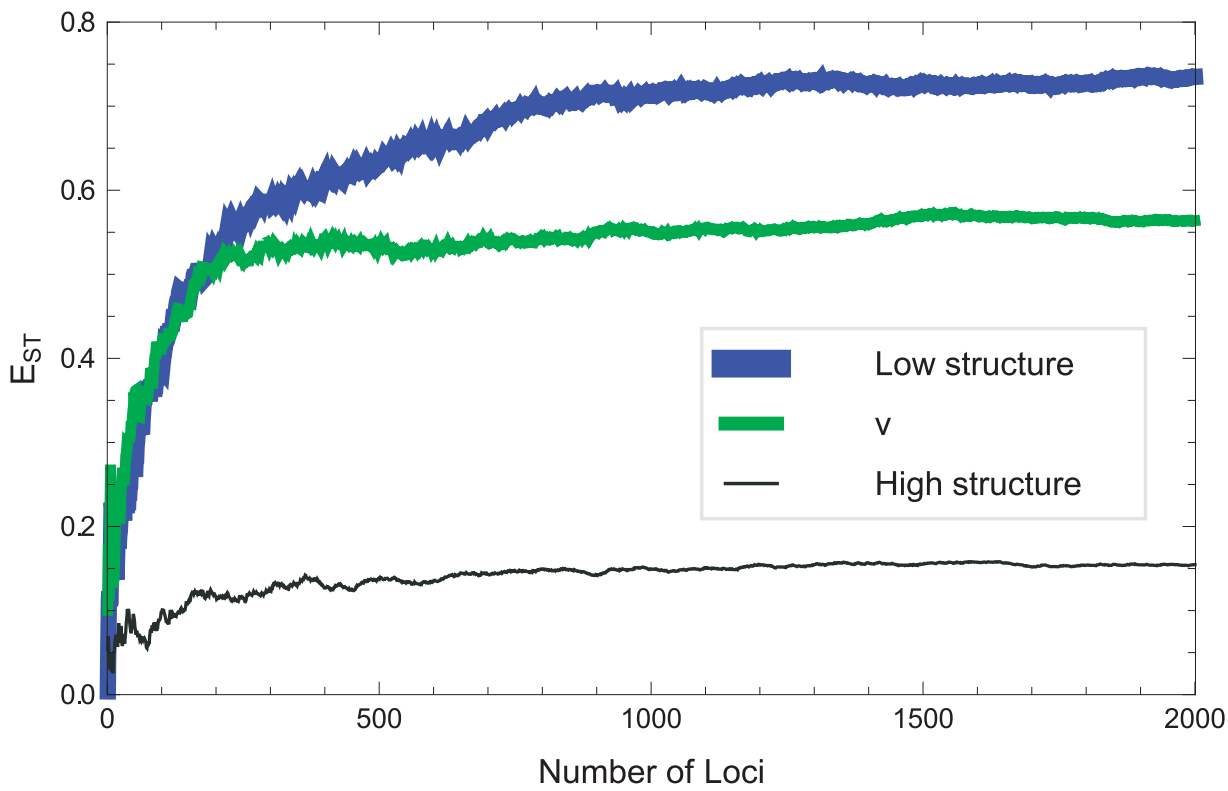

Fig 11. A numerical simulation of a model for $E_{\mathrm{ST}}$ for two structured populations (with $\boldsymbol{F}_{\mathrm{ST}}=0.05$ ). $E_{\mathrm{ST}}$ was computed using the formulation in Eq (2) of Materials and Methods.

doi:10.1371/journal.pone.0160413.g011 


\section{Supporting Information}

S1 Table. $\mathrm{E}_{\mathrm{ST}}$ min-max, $\mathrm{F}_{\mathrm{ST}}$ and $\mathrm{E}_{\mathrm{BT}}$ in $60 \mathrm{HGDP}$ population pairs ranked by $\mathrm{E}_{\mathrm{BT}}$. (TIF)

S1 Fig. $\mathrm{F}_{\mathrm{ST}}$ and $\mathrm{E}_{\mathrm{ST}}$ within 12 local regions. Calculated from a single pair of populations per region: Israel (Bedouins vs. Druze), Italy (North Italians vs. Tuscans), Mexico (Maya vs. Pima), France (Basque vs. French), Brazil (Karitiana vs. Surui), Pakistan (Burusho vs. Kalash) East Asia (Cambodian vs. Mongola), Europe (Russians vs. Sardinians), Oceania (Melanesians vs. Papuans), Pygmies (Biaka vs. Mbuti), Russia (Russians vs. Yakut) and Southern Africans (South African Bantu vs. San). The most obvious discrepancy between $\mathrm{F}_{\mathrm{ST}}$ and $\mathrm{E}_{\mathrm{ST}}$ is in Brazil, with a high $\mathrm{F}_{\mathrm{ST}}$ and moderate $\mathrm{E}_{\mathrm{ST}}$. The Druze and Bedouin of Israel live within a few hundred $\mathrm{km}$ of each other, speak the same language, and have the lowest $\mathrm{E}_{\mathrm{ST}}$ among these 12 pairs, yet have a somewhat higher $\mathrm{F}_{\mathrm{ST}}$ (several times higher than between the two Italian populations from Northern Italy and Tuscany and almost twice as high as between the French and Basques).

S2 Fig. Standard deviations (SD) of heterozygosity and pairwise genetic distances. From 660,755 SNPs in 53 HGDP populations. Excessive SD of genetic distance (blue) compared to SD of heterozygosity (red), as in the San and Naxi samples, implies the inclusion of relatives. (TIF)

S3 Fig. Individual standard deviations in six HGDP populations. Each column represents the SD between a single individual and all other samples in the given population. Tuscans $(\mathrm{n}=7)$, Italians $(\mathrm{n}=12)$, Naxi $(\mathrm{n}=8)$, Colombian $(\mathrm{n}=7)$, Surui $(\mathrm{n}=8)$, and Karitiana $(n=13)$. The "twin towers" in the Naxi batch are inferred to be a pair of close relatives in an otherwise panmictic population sample. These two individuals stick out like a sore thumb, while similarly related individuals are harder to identify among the Native American samples due to a higher base-level of structure in these population samples. (TIF)

S4 Fig. The SD of pairwise distance plotted against the SD of heterozygosity. Generated from the entire HGDP dataset (938 individuals from 53 populations). The red diagonal line represents the linear trend line of the standard deviation of heterozygosity. Populations above this line are inferred to have more genetic structure than expected from heterozygosity, implying that relatives may have been included in the samples. Native American populations, highlighted in light blue, appear to have moderate or moderately high levels of relatives included among their samples.

(TIF)

S5 Fig. Pairwise $\mathrm{F}_{S T}$ and $\mathrm{E}_{\mathrm{ST}}$ vs. geographic distance from the two Amazonian tribes to various global HGDP populations with increasing distance from the Amazon. (TIF)

S6 Fig. Neighbor-Joining trees of individual similarities. Generated from 660,755 SNPs. Individual branches are black, inter-population branches are red, and intra-population branches are blue. A. Complete trees. B. Zoom into trees with individual branches (black) removed. (TIF)

S7 Fig. Pairwise population distance charts. Each sample is represented by a red or blue string and each point on each string reflects distance between a pair of samples. Points that fall far below the rest are inferred to reflect close relatives.

(TIF) 
S8 Fig. Superimposed distance plots of Uygur and Adygei (top) and Surui and Maya (bottom). This is the same kind of plot as in S7 Fig, with each string representing a single individual. Despite a high $\mathrm{F}_{\mathrm{ST}}$ of $0.09\left(\mathrm{E}_{\mathrm{ST}}=0.52\right)$, some Mayan individuals (red) are genetically closer to some Surui individuals (blue) than to some fellow Mayan individuals $(\omega>0)$, presumably due to outbreeding (some Mayan individuals have significant European admixture, which increases distances among Mayans). There is no such overlap between Uygur (yellow) and Adygei (black) samples $(\omega=0)$ despite a much lower pairwise $\mathrm{F}_{\mathrm{ST}}$ of $0.02\left(\mathrm{E}_{\mathrm{ST}}=0.79\right)$. (TIF)

S9 Fig. Overview of $\mathrm{F}_{\mathrm{ST}}, \mathrm{E}_{\mathrm{ST}}$ and $\mathrm{E}_{\mathrm{BT}}$ among $60 \mathrm{HGDP}$ population pairs (660,755 SNPs). Negative $\mathrm{E}_{\mathrm{ST}} \mathrm{min}$ (yellow) and $\mathrm{E}_{\mathrm{ST}}$ mean (orange) would imply that close relatives were included among these samples. Of the 60 population pairs in the analysis, $12(20 \%)$ have negative $\mathrm{E}_{\mathrm{ST}}$ min values and 6 have negative $\mathrm{E}_{\mathrm{ST}}$ mean values. $\mathrm{E}_{\mathrm{ST}}$ median, $\mathrm{E}_{\mathrm{ST}} \mathrm{max}$, and $\mathrm{E}_{\mathrm{BT}}$ cover virtually the entire $0-1$ range with no negative values in these samples. The general trend is $\mathrm{F}_{\mathrm{ST}}$ $<\mathrm{E}_{\mathrm{ST}}$ min $<\mathrm{E}_{\mathrm{ST}}$ mean $<\mathrm{E}_{\mathrm{ST}}$ median $<\mathrm{E}_{\mathrm{ST}}$ max. $\mathrm{E}_{\mathrm{BT}}$ (gray) is usually somewhere between $\mathrm{E}_{\mathrm{ST} \text { - }}$ median (red) and $\mathrm{E}_{\mathrm{ST}} \mathrm{max}$ (black).

S10 Fig. Mean heterozygosity as a function of sample size. Heterozygosity in various HGDP populations with sample size increasing from 1 to 15 . All samples were included in populations with less than 15 samples (namely 7 in Colombians, 8 in Surui, and 13 in Karitiana).

S11 Fig. Pairwise $F_{S T}, E_{S T}$ and $E_{B T}$ as a function of sample size. Differentiation was estimated in two population pairs: French-Japanese and Surui-Karitiana, with population sample sizes ranging from $n=2$ to $n=8$. French-Japanese estimates were also taken at $n=15$ and $n=28$ due to their larger samples. $\mathrm{F}_{\mathrm{ST}}$ and $\mathrm{E}_{\mathrm{BT}}$ start at $\mathrm{n}=2 ; \mathrm{E}_{\mathrm{ST}}$ starts at $\mathrm{n}=3$, the minimal sample size for estimating the standard deviation of pairwise distances.

(TIF)

\section{Acknowledgments}

We thank Alan Templeton for helpful advice, Tat Dat Tran for assisting with one of the proofs in the appendix, Lior Lesch for software support, Sagi Abelson for help with the MATLAB script and two anonymous reviewers. KS wishes to acknowledge the Israel Science Foundation (grant 189/05) and the Beutler Fund at Rambam Medical Center for research support.

\section{Author Contributions}

Conceived and designed the experiments: YG.

Analyzed the data: YG OT.

Wrote the paper: YG.

Provided assessment and correction to the mathematics and crucial corrections to the interpretation and manuscript: SR. Refinement of the concept: KS. Guidance in relevant population genetics applications: KS. Revision of manuscript: KS.

\section{References}

1. Wright S (1978) Evolution and the Genetics of Populations. Vol. 4, Variability Within and Among Natural Populations. University of Chicago Press, Chicago. 
2. Nei M (1973) Analysis of gene diversity in subdivided populations. Proc Natl Acad Sci USA, 70, 33213323. PMID: 4519626

3. Weir BS, Cockerham CC (1984) Estimating F-Statistics for the Analysis of Population Structure. Evolution 38 (6): 1358.

4. Hudson RR, Slatkin M, Maddison WP (1992) Estimation of levels of gene flow from DNA sequence data. Genetics 132 (2), 583-589. PMID: 1427045

5. Tal O (2013) Two complementary perspectives on inter-individual genetic distance. Biosystems, 111: 18-36. doi: 10.1016/j.biosystems.2012.07.005 PMID: 22898797

6. Hedrick PW (2005) A standardized genetic differentiation measure. Evolution, 59, 1633-1638. PMID: 16329237

7. Jost $L$ (2008) $G_{S T}$ and its relatives do not measure differentiation. Mol Ecol, 17, 4015-4026. PMID: 19238703

8. Whitlock M (2011) G'st and D do not replace Fst. Mol Ecol 20:1083-109. doi: 10.1111/j.1365-294X 2010.04996.x PMID: 21375616

9. Lewontin RC (1972) The apportionment of human diversity. Evol Biol, 6, 381-98.

10. Witherspoon DJ, Wooding S, Rogers AR, Marchani EE, Watkins WS, Batzer MA, et al. (2007) Genetic Similarities Within and Between Human Populations. Genetics 176(1): 351-9. PMID: 17339205 doi: 10.1534/genetics.106.067355

11. Edwards AWF (2003) Human genetic diversity: Lewontin's fallacy, Bioessays, 25, 798-801. PMID: 12879450

12. Tal $\mathrm{O}$ (2012). The cumulative effect of genetic markers on classification performance: insights from simple models. J. Theor. Biol. 293 (January), 206-218.

13. Nelis M, Esko T, Mägi R, Zimprich F, Zimprich A, Toncheva D, et al. (2009) Genetic structure of Europeans: a view from the North-East. PLoS One 4: e5472. doi: 10.1371/journal.pone.0005472 PMID: 19424496

14. Patterson N, Price AL, Reich D (2006) Population Structure and Eigenanalysis. PLoS Genet 2: 20742093. doi: 10.1371/journal.pgen.0020190

15. Rosenberg NA, Pritchard JK, Weber JL, Cann HM, Kidd KK, e Zhivotovsky LA, et al. (2002) Genetic structure of human populations. Science 298: 2381-2385. PMID: 12493913

16. Cann HM, de Toma C, Cazes L, Legrand MF, Morel V, Piouffre $L$ (2002) A human genome diversity cell line panel. Science 296 (5566): 261-2. PMID: 11954565

17. Rosenberg NA (2006) Standardized subsets of the HGDP-CEPH Human Genome Diversity Cell Line Panel, accounting for atypical and duplicated samples and pairs of close relatives. Ann Hum Genet, 70: 841-847. PMID: 17044859

18. Jorde LB, Wooding SP (2004) Genetic variation, classification, and "race". Nat Genet, 36, S28-32. PMID: 15508000

19. Waples RS (1991) Definition of 'species' under the Endangered Species Act: Application to Pacific salmon. U.S. Department of Commerce NOAA Technical Memorandum, NMFS, F/NWC-194.

20. Darwin C (1871) The Descent of Man and Selection in Relation to Sex. London: John Murray.

21. Gao X, Martin ER (2009) Using allele sharing distance for detecting human population stratification. Hum Hered, 68, 182-191. doi: 10.1159/000224638 PMID: 19521100

22. Nakamura T, Shoji A, Fujisawa $H$, Kamatani $N(2005)$ Cluster analysis and association study of structured multilocus genotype data. J Hum Genet 50: 53-61. doi: 10.1007/s10038-004-0220-x PMID: 15696377 